HUTMP 94-B336

ESI 79 (1994)

UUITP $5 / 94$

UWThPh-1994-8

hep-th 9403066

\title{
Combinatorial Quantization of the Hamiltonian Chern-Simons Theory I
}

\author{
Anton Yu. Alekseev *† \\ Institute of Theoretical Physics, Uppsala University, \\ Box 803 S-75108, Uppsala, Sweden \\ HARALD Grosse $\ddagger$ \\ Institut für Theoretische Physik, Universität Wien, Austria \\ VOLKER SCHOMERUS $\S$ \\ Harvard University, Department of Physics \\ Cambridge, MA 02138, U.S.A.
}

March 9, 1994

\begin{abstract}
Motivated by a recent paper of Fock and Rosly [6] we describe a mathematically precise quantization of the Hamiltonian Chern-Simons theory. We introduce the Chern-Simons theory on the lattice which is expected to reproduce the results of the continuous theory exactly. The lattice model enjoys the symmetry with respect to a quantum gauge group. Using this fact we construct the algebra of observables of the Hamiltonian

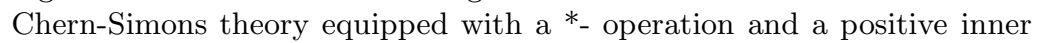
product.
\end{abstract}

\footnotetext{
* On leave of absence from Steklov Mathematical Institute, Fontanka 27, St.Petersburg, Russia; e-mail: alexeiev@rhea.teorfys.uu.se

${ }^{\dagger}$ Supported by Swedish Natural Science Research Council (NFR) under the contract F-FU 06821-304 and by the Federal Ministry of Science and Research, Austria

$\ddagger$ Part of project P8916-PHY of the 'Fonds zur Förderung der wissenschaftlichen Forschung in Österreich'; e-mail: grosse@pap.univie.ac.at

§upported in part by DOE Grant No DE-FG02-88ER25065; e-mail: vschomer@husc. harvard.edu
} 


\section{Introduction}

Quantization of the Chern-Simons theory in 3 dimensions has been attracting attention and efforts of many physicists and mathematicians during the last 5 years. The most spectacular results obtained in this way are the construction of knot invariants 13] and exact solution of $2+1$ dimensional gravity 27. Being a 3 dimensional topological field theory, the Chern-Simons model is intimately related to the Wess-Zumino-Novikov-Witten (WZNW) model of conformal field theory in 2 dimensions and to quantum groups (one may regard a quantum group as a 1 dimensional quantum system). Actually, it is this hierarchy of systems in different dimensions which makes the Chern-Simons theory solvable. The relation between CS and WZNW models allowed to evaluate partition functions and correlators [13] using the methods of conformal field theory. The importance of the CS theory is a motivation to look for different approaches. Among the others the perturbation theory [16] and exact evaluation of the functional integral by means of localization formulae 17] should be mentioned.

In this paper we develop an approach to the CS model based on its relation to the theory of quantum groups. One of the advantages of the quantum group approach is that we deal with finite dimensional objects only. As a consequence, one can represent the answers in terms of finite sums, whereas in other approaches the final result usually has an integral form. It makes the quantum group approach helpful in dealing with topology of 3D manifolds [24, 25, 26] and in knot theory [18], [23]. The main idea is to simulate the Chern-Simons theory on the lattice in such a way that partition functions and correlators of the lattice model coincide with those of the continuous CS model. It is important that the lattice model enjoys the gauge symmetry with respect to the quantum group. It is worth mentioning that a lattice simulation of the CS model has been suggested in [5]. The drawback of this model is the absence of gauge symmetry. The gauge symmetry may be restored if one uses the proper combinatorial description of the moduli space of flat connections [6].

Let us briefly characterize the content of each Section. In Section 2 we review the main facts concerning the CS theory in Hamiltonian approach and introduce the combinatorial (or lattice) description following [6]. Section 3 is devoted to the quantum gauge group in the lattice model. In Section 4 we generalize the concept of lattice gauge fields to the case of a quantum gauge group. The algebra of observables corresponding to the Hamiltonian CS theory appears in Section 5 equipped with a ${ }^{*}$-operation. We describe the Hermitian inner product in the algebra of observables and prove the positivity theorem in Section 6 . In section 7 we generalize the theory to weak quasi-Hopf algebras [2]. In this way we can deal with universal enveloping algebras at roots of unity by using a procedure called "truncation". The basic definition of weak quasi-Hopf algebras and the truncation are reviewed in section 7.1. An outlook at the end of the paper is devoted to the possible perspectives of the lattice approach. 
The basic technique which we use in this paper is the theory of quantum groups. Section 2 is supposed to play the role of the physicist oriented introduction. In the remaining part we assume that the reader is familiar with standard definitions and properties of such objects as co-multiplication and $R$-matrix.

We want to stress that the construction we propose in the following assigns a Chern-Simons type model to every pair of a marked Riemann surface and a weak quasi-Hopf algebra. The latter is not necessarily given by a truncated quantized enveloping algebra of some simple Lie algebra. It has been shown recently 8 that a weak quasi-Hopf symmetry can be constructed for every low dimensional quantum field theory. Combining this result with the considerations below, one assigns a Chern Simons type model to every low dimensional quantum field theory. It remains unclear whether these generalized Chern Simons theories are related to certain moduli spaces in the same way as standard Chern Simons theories are related to the moduli space of flat connections.

\section{Physical motivations}

In this paper we study the problem of quantization of the Chern-Simons theory within the Hamiltonian approach. The moduli space of flat connections on a Riemann surface appears as a phase space for this model [13]. Let us briefly remind the definition and general features of the CS system.

\subsection{Chern - Simons model}

The Chern-Simons theory is a gauge theory in 3 dimensions (in principle the CS term exists in any odd dimension). It is defined by the action principle

$$
C S(A)=\frac{k}{4 \pi} \operatorname{Tr} \int_{M}\left(A d A+\frac{2}{3} A^{3}\right) .
$$

Here $M$ is a 3-dimensional (3D) manifold, $k$ is a positive integer and the gauge field $A$ takes values in some semisimple Lie algebra $\mathcal{G}$

$$
A=A_{i}^{a} t^{a} d x_{i}
$$

The generators $t^{a}$ form a basis in $\mathcal{G}$ and satisfy the commutation relations

$$
\left[t^{a}, t^{b}\right]=f_{c}^{a b} t^{c}
$$

In this paper we concentrate on the very particular version of the CS theory when it has a Hamiltonian interpretation. Suppose that the manifold M locally looks like a cylinder $\Sigma \times R$ (Cartesian product of a Riemann surface $\Sigma$ and a segment of the real line). Then we may choose the direction parallel to the real line $R$ to be the time direction. Two space-like components of the gauge field $A$ become dynamical variables and we shall often denote by $A$ the two component 
gauge field on the surface $\Sigma$. As usual, the time-component $A_{0}$ becomes a Lagrangian multiplier. After the change of variables the action (2.1) acquires the form

$$
S=\frac{k}{4 \pi} \operatorname{Tr} \int\left(-A \partial_{0} A+2 A_{0} F\right) d t
$$

where the first term is just like $\int p d q$ and the second term introduces the first class constraint

$$
F=d A+A^{2}=0 .
$$

The first term in (2.4) determines the Poisson brackets (PB) of dynamic variables. In particular, the Poisson bracket of the constraints (2.5) may be easily calculated:

$$
\left\{F^{a}\left(z_{1}\right), F^{b}\left(z_{2}\right)\right\}=\frac{2 \pi}{k} f_{c}^{a b} F^{c}\left(z_{1}\right) \delta^{(2)}\left(z_{1}-z_{2}\right) .
$$

As one expects, the constraints (2.5) generate gauge transformations

$$
A^{g}=g^{-1} A g+g^{-1} d g \text {. }
$$

Thus, the phase space of the Hamiltonian CS theory is a quotient of the space $\Im$ of flat connections (2.5) over the gauge group $\Sigma G$ (2.7). We see that the moduli space (we shall often refer to the moduli space of flat connections as to the moduli space) appears to be a phase space of the CS theory on the cylinder. The action principle (2.4) provides canonical Poisson brackets on the moduli space. An efficient description of this $\mathrm{PB}$ was given in [6] (see also subsection $2.3)$.

\subsection{Wilson lines and marked points}

We continue our brief survey of the CS theory and consider possible observables. The CS model enjoys two important symmetries: gauge symmetry and the symmetry with respect to diffeomorphisms. The reparametrization symmetry appears due to the geometric nature of the action (2.1) which is written in terms of differential forms and automatically invariant with respect to diffeomorphisms of the manifold $M$. It is natural to require that the observables in the CS model respect the invariance properties of the theory. Some observables of this type may be constructed starting from the following data. Let us choose the closed contour $\Gamma$ in $M$ and a representation $I$ of the algebra $\mathcal{G}$. Apparently the following functional of the gauge field $\mathrm{A}$

$$
W_{I}(\Gamma)=\operatorname{Tr}_{I} P \exp \left(\int_{\Gamma} A^{I}\right)
$$

is invariant with respect to both gauge and reparametrization symmetries. Usually the contour $\Gamma$ is called a Wilson line and the expression (2.8) is called a Wilson line observable. The connection $A^{I}$ is equal to

$$
A^{I}=A^{a} T_{a}^{I}
$$


where matrices $T_{a}^{I}$ represent the algebra $\mathcal{G}$ in the representation $I$.

In the Hamiltonian formulation we may choose two special classes of Wilson lines: vertical and horizontal.

We call a Wilson line horizontal if it lies on an equal time surface. The observable corresponding to a horizontal Wilson line is a functional of the twodimensional gauge field and after quantization it becomes a physical operator.

The Wilson line is called vertical if the contour $\Gamma$ is parallel to the time axis. In the Hamiltonian picture we do not actually control the fact that vertical Wilson lines are closed. They come from the past, go through the zero-time surface and disappear in the future. The vertical Wilson line is characterized by the representation $I$ and the point $z$ where it intersects the Riemann surface $\Sigma$. The choice of the time axis produces a big difference in the role of horizontal and vertical Wilson lines in the theory. Vertical Wilson lines do not correspond to observables in the Hamiltonian formulation. Instead, they change the Hamiltonian system (2.4) so that both $\int p d q$ and the constraint get modified.

As a preparation for analyzing vertical Wilson lines let us remind the correspondence between co-adjoint orbits and representations of semi-simple Lie groups. Let $I$ be a representation of the Lie algebra $\mathcal{G}$ :

$$
\left[T_{a}^{I}, T_{b}^{I}\right]=f_{c}^{a b} T_{c}^{I} .
$$

One may ask the question what is the underlying classical system corresponding to the quantum algebra (2.10). The first idea is to simulate the commutation relations by the Poisson brackets:

$$
\left\{T_{a}, T_{b}\right\}=f_{c}^{a b} T_{c}
$$

where $T_{a}$ are commuting coordinates (on the dual space to the Lie algebra). It seems that the information about the representation disappeared in the formula (2.11). However, this is not quite true. The PB (2.11) is always degenerate. At this point it is useful to introduce a coordinate matrix

$$
T=T_{a} t^{a}
$$

where $t^{a}$ form a basis in the fundamental representation. In order to make the bracket (2.11) nondegenerate one should fix eigenvalues of the matrix $T$ :

$$
T=g^{-1} P g \text {. }
$$

A conjugation class $(2.13)$ is also called a co-adjoint orbit. Now we have a diagonal matrix $P$ which parametrizes the set of orbits. Quantization of a particular orbit leads to an irreducible representation of the Lie algebra. The highest weight $w_{I}$ of the representation $I$ represented as a diagonal matrix in the fundamental representation is related to $P$ by

$$
w_{I}=P-\rho,
$$


where $\rho$ is a half sum of positive roots of $\mathcal{G}$.

The information about the bracket (2.11) on the orbit may be encoded in the action

$$
S_{I}(g)=\operatorname{Tr} \int P d g g^{-1} .
$$

The action (2.15) is called a geometric action because it originates from the method of geometric quantization. The family of systems (2.15) is parametrized by the set of representations of $\mathcal{G}$ which may be obtained upon quantization.

The essence of the quantization procedure for the action (2.15) is the following formula for a Wilson line observable:

$$
W_{I}=\int e^{i\left(S_{I}(g)+\int A_{0}^{a} T^{a} d t\right)} D g .
$$

The ordered exponent and trace are provided by the functional integral automatically.

Using the formula (2.16), one may treat the CS correlator with $n$ vertical Wilson lines inserted

$$
Z_{k}\left(I_{1}, \ldots, I_{n}\right)=\int D A e^{i C S(A)} W_{I_{1}} \ldots W_{I_{n}}
$$

as an expression where the gauge field is still classical, whereas some modes corresponding to the matrices $T_{i}$ are already quantized. The original functional integral would be

$$
Z=\int D A D g_{1} \ldots D g_{n} e^{i S^{t o t}}
$$

The action $S^{\text {tot }}$ is defined by the formula

$$
S^{\text {tot }}=C S(A)+\sum_{i=1}^{n}\left(S_{I_{i}}\left(g_{i}\right)+\operatorname{Tr} \int A_{0}\left(z_{i}\right) T_{i} d t\right)
$$

Here the first term coincides with the standard Chern-Simons action, the second term consists of two parts. The first part collects auxiliary geometric actions for each Wilson line, the second part represents contributions of the Wilson lines into the CS partition function (2.17).

We have reformulated the Hamiltonian Chern-Simons model with vertical Wilson lines as a theory of the 2D gauge field $A$ interacting with a set of finite dimensional systems with coordinates $T_{i}$ localized at the points $z_{i}$. As in the case of the pure CS theory, the Hamiltonian (2.19) is equal to zero. The action of the modified system may be rewritten as

$$
S^{\text {tot }}=\operatorname{Tr}\left(-\frac{k}{4 \pi} \int A \partial_{0} A+\sum_{i=1}^{n} P_{i} d g_{i} g_{i}^{-1}\right)+\operatorname{Tr} \int A_{0}\left(\frac{k}{2 \pi} F+\sum_{i=1}^{n} T_{i} \delta\left(z-z_{i}\right)\right) .
$$


The first term in $(2.20)$ is of the type $\int p d q$ of the Hamiltonian system. It is responsible for the Poisson brackets of dynamical variables. The second term gives the modified constraint

$$
\Phi(z)=F+\frac{2 \pi}{k} \sum_{i=1}^{n} T_{i} \delta\left(z-z_{i}\right)=0 .
$$

The constraints (2.21) satisfy the same algebra (2.6) as in the pure CS theory. They generate gauge transformations for the gauge field $A$ and conjugations for the variables $T_{i}$ :

$$
A^{g}=g^{-1} A g+g^{-1} d g \quad, \quad T_{i}^{g}=g\left(z_{i}\right)^{-1} T_{i} g\left(z_{i}\right) .
$$

Now the phase space of the Hamiltonian Chern-Simons theory with vertical Wilson lines may be described. First we mark $n$ points $\left\{z_{i}\right\}$ on the Riemann surface $\Sigma$ of genus $g$. Each point is equipped with a representation $I_{i}$ of the algebra $\mathcal{G}$ (and corresponding orbit $\left.O_{i}\right)$. One can choose a subspace $\Im\left(I_{1}, \ldots, I_{n}\right)$ defined by the constraint (2.21) in the Cartesian product $C_{g} \times O\left(I_{1}\right) \times \ldots \times O\left(I_{N}\right)$ of the space of all connections $C_{g}$ on the Riemann surface and co-adjoint orbits attached to the marked points. The subspace $\Im\left(I_{1}, \ldots, I_{n}\right)$ is a natural analogue of the space of flat connections. It is invariant with respect to gauge transformations (2.22). The quotient of $\Im\left(I_{1}, \ldots, I_{n}\right)$ by the action of the gauge group may be called the moduli space $\mathcal{M}_{g, n}(\mathcal{G})$ of flat connections on the Riemann surface of genus $g$ with $n$ marked points. The moduli space $\mathcal{M}_{g, n}(\mathcal{G})$ inherits the Poisson structure from the CS theory. This structure is the subject of the next subsection.

Let us finish by a short remark concerning the structure of $\mathcal{M}_{g, n}(\mathcal{G})$ and the theory of orbits. Choosing a small loop $\Gamma_{i}$ surrounding the marked point $z_{i}$, one can define the monodromy matrix (or parallel transport) $M_{i}$ along this path. It is easy to check that if $A$ and $\left\{T_{i}\right\}$ satisfy (2.21), the monodromy matrix $M_{i}$ may be diagonalized by conjugation of the exponent of $P_{i}$

$$
M_{i}=h^{-1} \exp \left(\frac{2 \pi}{k} P_{i}\right) h .
$$

In the quantum case there is a one-loop correction in this formula which adds the dual Coxeter number $h^{*}$ to the parameter $k$. The correct quantum version of (2.23) reads as

$$
M_{i}=h^{-1} \exp \left(\frac{2 \pi}{k+h^{*}} P_{i}\right) h .
$$

Thus, the monodromy matrix belongs to the exponentiated orbit assigned to the corresponding marked point. Formulae (2.23) and (2.24) characterize elementary monodromies. They will be quite helpful for quantization of $\mathcal{M}_{g, n}(\mathcal{G})$. 


\subsection{Combinatorial description and Exchange relations}

In the previous subsection we have found that the moduli space $\mathcal{M}_{g, n}(\mathcal{G})$ appears as a phase space in the Chern-Simons theory with vertical Wilson lines. In order to quantize the theory we need the Poisson structure on $\mathcal{M}_{g, n}(\mathcal{G})$. In principle one may proceed starting from the $2 \mathrm{D}$ gauge fields with the Poisson bracket

$$
\left\{A_{i}^{a}\left(z_{1}\right), A_{j}^{b}\left(z_{2}\right)\right\}=-\frac{2 \pi}{k} \delta^{a b} \epsilon_{i j} \delta^{(2)}\left(z_{1}-z_{2}\right) .
$$

The quantization of the relations (2.25) is straightforward

$$
\left[A_{i}^{a}\left(z_{1}\right), A_{j}^{b}\left(z_{2}\right)\right]=-\frac{2 \pi}{k} \delta^{a b} \epsilon_{i j} \delta^{(2)}\left(z_{1}-z_{2}\right) .
$$

Observables may be described as gauge invariant functionals of $A$, where the constraint (2.21) is imposed. In this approach we deal with the representations of the infinite dimensional algebra $(2.26)$ and construct the quantum mechanics corresponding to the CS system starting from the field theory of the gauge field A. It is a motivation to look for another approach. A recent progress [6] in this direction allows to reformulate the problem. The idea is to simulate the Riemann surface by the oriented fat graph drawn on it. Dealing with a fat graph one uses ribbons instead of strings. It means that the cyclic order of links incident to a given vertex is fixed.

Suppose that we have a gauge field $A$ on the Riemann surface and a graph drawn on it. We assume that the surface is divided by the graph into plaquettes so that any plaquette is contractible. The graph should be chosen in such a way that the number of marked points inside each plaquette does not exceed one. The gauge field defines a parallel transport along each link of the graph. Let us enumerate the vertices of the graph by letters $x, y, z \ldots$ and the links by $i, j, k \ldots$ It is convenient to introduce notations $s(i)$ and $t(i)$ for the end-points of the link $i$. The parallel transport corresponding to this link may be written as an ordered exponent

$$
U(i)=\operatorname{Pexp}\left(\int_{s(i)}^{t(i)} A\right)
$$

As for the Wilson lines, one may introduce the set of matrices $U^{I}(i)$

$$
U^{I}(i)=\operatorname{Pexp}\left(\int_{s(i)}^{t(i)} A^{I}\right)
$$

using the gauge field in different representations (2.9).

Some information about the connection $A$ is encoded in the link variables (2.27). The question is whether this information is sufficient to reconstruct the moduli space $\mathcal{M}_{g, n}(\mathcal{G})$ ? The answer is obviously positive. To recover the 
moduli space we should factorize over residual gauge transformations and take into account the flatness condition. Gauge transformations act on the graph connections (2.27) as follows

$$
U(i)^{h}=h(s(i))^{-1} U(i) h(t(i)) .
$$

It is remarkable that the gauge group becomes effectively finite dimensional because only values of $h$ in graph vertices enter into (2.29).

The condition of flatness may be simulated using the properties of the monodromy from the previous Subsection. We form the monodromy for each plaquette and constrain it by the condition (2.23). If there is no marked point inside the plaquette, the monodromy is simply equal to the identity.

It is proved [6] that factorizing the space of flat graph connections over graph gauge transformations one obtains the same space $\mathcal{M}_{g, n}(\mathcal{G})$ ! Moreover, the Poisson structure on the space of graph connections leading to the standard Poisson structure on $\mathcal{M}_{g, n}(\mathcal{G})$ is known. In this approach the moduli space is represented as a quotient of the finite dimensional space over the finite dimensional group. This is the reason to call it combinatorial description of the moduli space.

One may think that the Poisson brackets for graph connections are defined uniquely since $U(i)$ are functionals of the gauge field $A$ and the Poisson brackets for $A$ are fixed by (2.25). In fact, this is not correct. The reason is the $\delta$-function singularity in the brackets (2.25). Calculating the PB of two matrices $U(i)$ and $U(j)$, where the links $i$ and $j$ have at least one common end-point, one has to resolve the singularity appearing at this very point. There is no canonical way of resolution. In general the Jacobi identity breaks down after regularization. It means that one can't construct the Poisson bracket applicable for both local gauge fields $A(x)$ and arbitrary link variables $U(i)$. However, it is possible to introduce meaningful Poisson brackets for link variables $U(i)$. So, the brackets for $A(x)$ and for $U(i)$ are to some extent independent from each other. One calls them consistent if they give the same answer for Poisson brackets of gauge invariant variables. In other words, $A$ - and $U$-bracket may differ in nonphysical sector but they coincide when we restrict ourselves to physical gauge invariant observables.

In order to simplify the analysis of the $U$-bracket, we remind here some standard definitions and properties of quadratic Poisson brackets and Exchange algebras.

Suppose that we have a Poisson bracket defined on the matrix group. The simplest form of such bracket is quadratic in matrix elements

$$
\left\{U^{1}, U^{2}\right\}=U^{1} U^{2} r
$$

where we have used tensor notations

$$
U^{1}=U \otimes i d, U^{2}=i d \otimes U
$$


and the matrix $r$ is defined in the tensor product of two vector spaces. To ensure the Jacobi identity, the following condition on the matrix $r$ must be satisfied

$$
\left[r_{12}, r_{23}\right]+\left[r_{12}, r_{13}\right]+\left[r_{13}, r_{23}\right]=0 .
$$

The constraint (2.32) is the classical Yang-Baxter equation. Fortunately, for any simple Lie algebra we know two solutions to the very complicated equation (2.32). Usually they are called $r$ and $r^{\prime}$ and look as follows

$$
\begin{aligned}
& r=\sum_{i} h^{i} \otimes h^{i}+2 \sum_{\alpha} t^{\alpha} \otimes t^{-\alpha}, \\
& r^{\prime}=\sum_{i} h^{i} \otimes h^{i}+2 \sum_{\alpha} t^{-\alpha} \otimes t^{\alpha} .
\end{aligned}
$$

Here the sum in the first term runs over the set of simple roots and in the second over the set of positive roots.

The Poisson algebra (2.30) may be quantized if one knows a one parameter family of solutions $R(h)$ of the quantum Yang-Baxter equation

$$
R_{12} R_{13} R_{23}=R_{23} R_{13} R_{12}
$$

with given asymptotics

$$
R=1+h r+\ldots .
$$

Using $R(h)$ we quantize the bracket (2.30) in the following way

$$
U^{1} U^{2}=U^{2} U^{1} R
$$

We shall denote solutions of the quantum Yang-Baxter equation corresponding to the classical $r$-matrices (2.33,2.34) by $R$ and $R^{\prime}$. It is assumed everywhere in the text that both of them depend on the deformation parameter $h$. It is worth mentioning that

$$
R^{\prime}=P R P,
$$

where $P$ is a permutation matrix in the tensor product of two copies of the same vector space. It is important that along with $R$ - matrices in the fundamental representation there exists a family of $R$ - matrices parametrized by pairs $(I, J)$ of finite dimensional representations of $\mathcal{G}$ so that $R^{I J}$ act in the tensor product of the representation spaces of $I$ and $J$ and equation (2.35) holds for any triple of representations $(I, J, K)$ corresponding to the indices 1,2,3.

In the class of regularizations suggested in [6] the Poisson brackets of graph connections are quadratic. Let us first describe these brackets and then quantize.

To fix Poisson brackets for graph connections one must choose some particular regularization of the singularity in (2.25). In practice it means that each vertex should be equipped with a classical $r$-matrix (in the quantum case quantum $R$ - matrix) from a certain equivalence class. Roughly speaking the 
class is defined by the choice of the deformation parameter $h$. One also should fix a linear order of incident links at each vertex in addition to the natural cyclic order. The latter may be done by putting a little eyelash at each graph vertex. The eyelash determines from where we enumerate links coming to the vertex. Concerning the choice of $r$-matrices, we shall restrict ourselves to the particular regularization so that the $r$-matrices assigned to all vertices of the graph coincide.

There are three rules which determine the structure of the Poisson algebra of graph connections:

1) The Poisson bracket of any matrix elements of two parallel transport matrices corresponding to links which have no common end-points vanishes:

$$
\left\{U(i)^{1}, U(j)^{2}\right\}=0
$$

This condition brings locality into the definition of the Poisson bracket. Indeed, the original bracket for 2-dimensional connections had a support at coincident points. If the links have no common end-points, they do not intersect at all. It means that the bracket of the corresponding matrix elements should vanish if we want to reproduce the continuous theory.

2) For the matrix elements of the same matrix we have

$$
\left\{U(i)^{1}, U(i)^{2}\right\}=\frac{2 \pi}{k}\left(r U(i)^{1} U(i)^{2}-U(i)^{1} U(i)^{2} r^{\prime}\right) .
$$

3) If the links have one common end-point, the Poisson bracket acquires the form

$$
\left\{U(i)^{1}, U(j)^{2}\right\}=\frac{2 \pi}{k} U(i)^{1} U(j)^{2} r
$$

if the link $i$ is elder than the link $j$ in the clock-wise order starting from the eyelash (we can express it as $i<j$ ) and

$$
\left\{U(i)^{1}, U(j)^{2}\right\}=-\frac{2 \pi}{k} U(i)^{1} U(j)^{2} r^{\prime}
$$

otherwise.

From the definitions of the Poisson algebra on the space of graph connections we learn that the deformation parameter in this theory is equal to $h=\frac{2 \pi}{k}$. Actually, this formula is correct only semiclassicaly. In the Hamiltonian formulation the Chern-Simons integral is Gaussian. So, we should worry only about possible one-loop corrections. Depending on the definition of the integration measure in the Chern-Simons functional integral, one gets different results for the renormalized value of the parameter $k$. In the standard scheme which we follow in this paper, $k$ receives a one loop correction equal to the dual Coxeter number $k \rightarrow k+h^{*}$. So, the correct value of the deformation parameter is $h=\frac{2 \pi}{k+h^{*}}$. 
Another one loop effect which shows up in the Chern-Simons theory is the framing anomaly (see e.g. 20]). It appears that in order to define the renormalized CS model one should fix a frame in the 3D manifold $M$ and replace Wilson lines by ribbons. Physical correlation functions depend on the choice of the framing. The exact form of this dependence is governed by the framing anomaly. In the Hamiltonian version of the CS theory we always have a prefered framing invariant with respect to shifts along the time axis. We can stick to this framing from the very beginning and in this way we do not trace the framing anomaly in the Hamiltonian approach.

At this point we want to stress that the information about the one loop correction of $k$ is the only external data which we bring into the scheme of combinatorial quantization. Further steps are quite independent of the Lagrangian formulation of the theory and give a selfconsistent approach to the Chern-Simons model.

The relations (2.41) and (2.42) are written for the situation when both oriented links point towards their common end-point. All the other relations may be derived following the rules, if we assume that for the same link taken with two different orientations the corresponding link variables are inverse to each other:

$$
U(i) U(-i)=U(-i) U(i)=1
$$

For example, if $i<-j$ and the link $j$ starts from the common end-point the Poisson bracket (2.42) will be modified

$$
\left\{U(i)^{1}, U(j)^{2}\right\}=-U(j)^{2} r U(i)^{1} .
$$

So we have described the Poisson algebra for the gauge field on the graph. Now the problem of quantization is in order. As we discussed, quadratic $r$ matrix Poisson brackets admit straightforward quantization. Let us list the corresponding quantum formulae:

$$
U(i)^{1} U(j)^{2}=U(j)^{2} U(i)^{1}
$$

for links $i$ and $j$ which have no intersection points;

$$
U(i)^{1} U(i)^{2}=R U(i)^{2} U(i)^{1}\left(R^{\prime}\right)^{-1},
$$

for the matrix elements of the same matrix;

$$
U(i)^{1} U(j)^{2}=U(j)^{2} U(i)^{1} R
$$

for two links which have a common target when $i<j$ and

$$
U(i)^{1} U(j)^{2}=U(j)^{2} U(i)^{1}\left(R^{\prime}\right)^{-1}
$$

for $i>j$. The quantum algebra defined by the relations $2.45-2.48$ may be treated as a noncommutative analogue of a lattice gauge field. As we see, the 
lattice emerges naturally in this approach. Moreover, we do not know how to get rid of it in this type of noncommutative gauge models. It may happen that the lattice formulation is dictated by the noncommutative nature of the gauge field algebra.

The next question concerns the generalization of the gauge symmetry to the noncommutative gauge theory. As in any lattice gauge theory, gauge transformations act at the lattice vertices:

$$
U(i)^{h}=h(s(i))^{-1} U(i) h(t(i))
$$

It is easy to check that these transformations do not preserve the exchange relations for $U$ 's unless we assume that $h$ entering $(2.49)$ are also noncommutative. More exactly, $h(x)$ and $h(y)$ commute for $x$ and $y$ being different vertices

$$
h(x)^{1} h(y)^{2}=h(y)^{2} h(x)^{1}
$$

but form a (not co-commutative) Hopf algebra at each vertex.

The fact that the noncommutative gauge algebra is invariant with respect to the quantum group valued gauge transformations may be expressed also in the following way. We can treat matrix indices of $U(i)$ as indices of the fundamental representation of the corresponding quantized universal enveloping algebra. If we consider the Chern-Simons theory of $\mathcal{G}$-valued gauge fields with coefficient $k$ in the action, the quantum symmetry is an algebra $U_{q}(\mathcal{G})$ for $q$ being equal to $\exp \left(2 \pi i /\left(k+h^{*}\right)\right)$.

Here one can change the point of view and try to construct the noncommutative gauge fields starting from some symmetry algebra placed at the lattice sites. It may be a quantum group but one can choose also some other symmetry algebra. In particular, choosing the nondeformed Lie algebra $\mathcal{G}$ one should recover the standard two dimensional lattice gauge theory. In this paper we explore the approach based on the symmetry algebra and find that the gauge theory may be reconstructed if the symmetry algebra is endowed with co-multiplication. The latter means that one can construct tensor products of representations of the symmetry algebra and decompose them into irreducible ones. In more mathematical language it means that the symmetry algebra is considered as a Hopf algebra (see Section 3) or as a quasi-Hopf algebra (see Section 7).

Down to earth, along with the matrix $U(i)$ in the fundamental representation of the symmetry algebra we introduce a bunch of matrices for any representation as in formula (2.28). It is not difficult to generalize quadratic exchange relations for this case. For example, instead of (2.47) we get

$$
U^{I}(i)^{1} U^{J}(j)^{2}=U^{J}(j)^{2} U^{I}(i)^{1} R^{I J} .
$$

Matrices $U^{I}(i)$ are not independent. They form a closed algebra so that a product of any two matrix elements may be decomposed into a linear combination of matrix elements. Formula (2.43) gives a simplest example of such relations. The 
algebra of matrix elements of $U^{I}(i)$ is closely related to the algebra of functions on the finite dimensional group $G$. The structure constants $[\ldots]$ which appear in the decomposition

$$
g_{a b}^{I} g_{c d}^{J}=\sum \overline{\left[\begin{array}{lll}
I & J & K \\
a & c & e
\end{array}\right]^{\alpha}} g_{e f}^{K}\left[\begin{array}{lll}
I & J & K \\
b & d & f
\end{array}\right]^{\alpha}
$$

for the matrix elements of $G$ are usually called Clebsch-Gordan coefficients. They are defined as invariant tensors in the triple tensor product of representations $I, J$ and $K$ and parametrized by an integer $\alpha$. In formula (2.52) the summation over $e, f, K$ and $\alpha$ is assumed. Generalizing such relations, the Hopf (or quasi-Hopf) structure of the symmetry algebra enters in practice. The algebra of $U^{I}(i)$ looks exactly like (2.52) but the structure constants $C$ must be replaced by the Clebsch-Gordan coefficients for the corresponding quantum algebra.

Let us give a simple example to clarify the definition of the exchange algebra for graph connections. We pick up an elementary plaquette on the Riemann surface and enumerate the links from 1 to $s$ in the counter clock-wise order. It is convenient to choose the orientations so that all the arrows are also directed against the clock rotation. We choose the eyelashes at all vertices to be directed inside the plaquette. Under these conditions link variables $U(1) \ldots U(s)$ may be treated independently of the rest of the graph. The corresponding exchange algebra looks as follows:

$$
U(i)^{1} U(i)^{2}=R U(i)^{2} U(i)^{1}\left(R^{\prime}\right)^{-1}
$$

for any link $i$;

$$
U(i)^{1} U(i+1)^{2}=U(i+1)^{2} R^{-1} U(i)^{1},
$$

where we assume that by definition $U(s+1)=U(1)$. As usual, the matrix elements of $U(i)$ and $U(j)$ commute if $i$ and $j$ have no common end-points.

Actually, the graph connection algebra does not know if there is a piece of surface inside the plaquette or, perhaps, there is a hole there and the links which surround the plaquette lie on the boundary of the surface. So one can try to describe the boundary in the Chern-Simons theory using the algebra (2.53,2.54). The theory living on the boundary is the chiral WZNW model. It is not topological and we cannot hope to describe it in an adequate way using our rough lattice approximation. On the other hand, if one increases the number of lattice sites so that the distance between them becomes smaller and smaller, the lattice exchange algebra (2.53,2.54) admits a nice continuous limit. Under certain assumptions it is possible to prove that this continuous limit coincides with the Kac-Moody algebra which governs the WZNW model assigned to the boundary. It was the reason to introduce the lattice exchange algebra (2.53,2.54) as lattice current algebra in 19 .

So, for an appropriate choice of ciliation the graph connection exchange algebra includes lattice Kac-Moody algebras for particular plaquettes as its subalgebras. It is one extra check of consistency of our lattice model. 
We have described the basic structures that we are going to investigate in this paper. Let us remark that the lattice simulations of the Chern-Simons theory and of the moduli space of flat connections are expected to give exact results because the Poisson structure and the phase space may be reproduced exactly on the semiclassical level. The quantum theory on the graph appears to be a lattice gauge theory associated to the quantum group. This theory enjoys the quantum gauge symmetry and this is the main difference between our model and the model [5] where the relations (2.47 2.48) are replaced by the commutative relation of the type (2.45). It is remarkable that the quantization of the Chern-Simons theory leads to quadratic algebra which uses R-matrices as structure constants. It makes the theory efficiently finite dimensional and this is the reason to call this approach combinatorial quantization of the Chern-Simons model.

Now we change the language to a more mathematical one and turn to the systematical treatment of the algebra of observables of the Hamiltonian ChernSimons theory.

\section{The algebra $\mathcal{G}$ of gauge transformations}

This section is devoted to a precise formulation of local gauge symmetries on the graph or lattice. Gauge transformations $\xi$ will be assigned to the vertices of the graph. The algebra $\mathcal{G}$ of all gauge transformations comes equipped with the structure of a ribbon Hopf-*-algebra.

\subsection{The algebraic structure of $\mathcal{G}$}

To be specific, we consider a graph $G$ formed by the edges and vertices of a triangulation of a given oriented Riemann surface $\Sigma$. For every oriented link $i$ of $G$ there is an oriented link $-i$ which has opposite orientation. The set of oriented links $i,-i, j,-j, k,-k, \ldots$ will be denoted by $L$. For elements in the set $S$ of vertices we use the letters $x, y, z$. We introduce the map $t: L \mapsto S$ such that $t(i)=x$, if the oriented link $i$ points towards the vertex $x$.

We describe the local gauge symmetry by assigning a ribbon Hopf-*-algebra $\mathcal{G}_{x}$ to every vertex $x \in S$. Ribbon Hopf-algebras were introduced in [28]. Their definition is based on the algebraic structure of quasitriangular Hopf-*-algebras, so that the algebras $\mathcal{G}_{x}$ come equipped with a co-unit $\epsilon_{x}$, a co-product $\Delta_{x}$, an antipode $\mathcal{S}_{x}$ and an $R$-matrix $R_{x}$. While we assume the reader to be familiar with the defining properties of a quasitriangular Hopf algebra, we want to make some more detailed remarks on the $*$-operation. In a Hopf-*-algebra co-product, co-unit and antipode have to be consistent with the conjugation $*$. In detail this implies that $\epsilon_{x}$ and $\Delta_{x}$ are ${ }^{*}$-homomorphisms, i.e.

$$
\epsilon_{x}\left(\xi^{*}\right)=\overline{\epsilon_{x}(\xi)} \quad, \quad \Delta_{x}\left(\xi^{*}\right)=\Delta_{x}(\xi)^{*} .
$$


Since $\Delta_{x}(\xi)$ is an element of $\mathcal{G}_{x} \otimes \mathcal{G}_{x}$, the second equation requires an action of $*$ on $\mathcal{G}_{x} \otimes \mathcal{G}_{x}$. This action is not unique. One can either define $(\xi \otimes \eta)^{*}=\xi^{*} \otimes \eta^{*}$ or (cp. [2])

$$
(\xi \otimes \eta)^{*}=\eta^{*} \otimes \xi^{*}
$$

Throughout this paper we will consider the second case (3.1). The main reason is that this type of $*$-operation appears in many interesting examples, e.g. in $U_{q}\left(s l_{2}\right), q^{p}=1$. Readers interested in the first case can easily rewrite everything below. The construction of a scalar product on the space of physical states simplifies dramatically.

It is consistent to demand that the antipode $\mathcal{S}_{x}$ is a ${ }^{*}$-anti-homomorphism 12.

$$
\mathcal{S}_{x}\left(\xi^{*}\right)=\mathcal{S}_{x}(\xi)^{*}
$$

In a quasi-triangular Hopf-*-algebra, unitarity of the $R$-matrix $R_{x}=\sum_{\sigma} r_{x \sigma}^{1} \otimes$ $r_{x \sigma}^{2}$,

$$
R_{x}^{*}=\sum_{\sigma} r_{x \sigma}^{2 *} \otimes r_{x \sigma}^{1 *}=R_{x}^{-1}
$$

is assumed to hold. Again these properties can easily be checked in the example $U_{q}\left(s l_{2}\right), q^{p}=1$.

Now let us proceed towards a description of ribbon Hopf-*-algebras [28]. Given the $R_{x}$-element, we build $u_{x} \in \mathcal{G}_{x}$ from its components,

$$
u_{x}=\sum_{\sigma} \mathcal{S}_{x}\left(r_{x \sigma}^{2}\right) r_{x \sigma}^{1}
$$

The standard properties of the element $u_{x}$ are

$$
\begin{gathered}
u_{x} \mathcal{S}_{x}^{-1}(\xi)=\mathcal{S}_{x}(\xi) u_{x} \quad, \quad u_{x}^{*}=u_{x}^{-1} \\
\Delta_{x}\left(u_{x}\right)=\left(u_{x} \otimes u_{x}\right)\left(R_{x}^{\prime} R_{x}\right)^{-1}=\left(R_{x}^{\prime} R_{x}\right)^{-1}\left(u_{x} \otimes u_{x}\right) .
\end{gathered}
$$

Moreover, the combination $u_{x} \mathcal{S}_{x}\left(u_{x}\right)$ is in the center of $\mathcal{G}_{x}$. To obtain a ribbon Hopf-*-algebra we postulate the existence of a central "square root" $v_{x}$ of this element which is supposed to obey

$$
\begin{aligned}
v_{x}^{2}=u_{x} \mathcal{S}_{x}\left(u_{x}\right) \quad, \quad \mathcal{S}_{x}\left(v_{x}\right)=v_{x} \quad, \quad \epsilon_{x}\left(v_{x}\right)=1, \\
v_{x}^{*}=v_{x}^{-1} \quad, \quad \Delta_{x}\left(v_{x}\right)=\left(R_{x}^{\prime} R_{x}\right)^{-1}\left(v_{x} \otimes v_{x}\right) .
\end{aligned}
$$

Such elements are known to exist for the quantized universal enveloping algebras of all simple Lie algebras 28].

One could demand that all the algebras $\mathcal{G}_{x}$ are isomorphic as Hopf algebras. But this is more than we need. To prepare for a weaker statement let us recall the notion of twist equivalence. $\mathcal{G}_{x}$ is said to be twist equivalent to another ribbon 
Hopf- ${ }_{\text {-algebra }} \mathcal{G}_{*}$ with co-unit $\epsilon_{*}$, co-product $\Delta_{*}$, antipode $\mathcal{S}_{*}, R$-matrix $R_{*}$ and ribbon element $v_{*}$, if there is a ${ }^{*}$-isomorphism $\iota_{x}: \mathcal{G}_{x} \mapsto \mathcal{G}_{*}$ such that

$$
\begin{aligned}
\epsilon_{x}(\xi)=\epsilon_{*}\left(\iota_{x}(\xi)\right) & , \quad \iota_{x}\left(\mathcal{S}_{x}(\xi)\right)=\mathcal{S}_{*}\left(\iota_{x}(\xi)\right), \\
\left(\iota_{x} \otimes \iota_{x}\right)\left(\Delta_{x}(\xi)\right) & =F_{x}^{-1} \Delta_{*}\left(\iota_{x}(\xi)\right) F_{x}, \\
\left(\iota_{x} \otimes \iota_{x}\right)\left(R_{x}\right)={F^{\prime}}_{x}^{-1} R_{*} F_{x} & , \quad \iota_{x}\left(v_{x}\right)=v_{*}
\end{aligned}
$$

holds for all $\xi \in \mathcal{G}_{x}$. Here $F_{x} \in \mathcal{G}_{*} \otimes \mathcal{G}_{*}$ is unitary, i.e. $F_{x}^{*}=F_{x}^{-1}$, and $F_{x}^{\prime}$ denotes the same element with exchanged components in the tensor product. If we would restrict ourselves to $F_{x}=e \otimes e$, we would end up with the usual notion of isomorphic Hopf-*-algebras. For the moment we assume that both coproducts $\Delta_{x}, \Delta_{*}$ are co-associative. This amounts to a severe restriction on $F_{x}$. However one can check that $F_{x}=R_{*}^{-1}$ is related to a non-trivial twist, which gives $\left(\iota_{x} \otimes \iota_{x}\right)\left(R_{x}\right)=R_{*}^{\prime-1}$. Using this weak notion of equivalence of Hopf-*algebras it is natural to demand that all the algebras $\mathcal{G}_{x}$ are twist equivalent to the same ribbon Hopf-*-algebra $\mathcal{G}_{*}$. In other words we assume the algebras $\mathcal{G}_{x}$ of local gauge transformations to be pairwise twist-equivalent. Let us mention that the element $u_{x}$ introduced above is independent of the twist in the sense that

$$
\iota_{x}\left(u_{x}\right)=u_{*} .
$$

The full gauge symmetry $\mathcal{G}$ is obtained as a product over all local gauge symmetries $\mathcal{G}_{x}$,

$$
\mathcal{G}=\bigotimes_{x \in S} \mathcal{G}_{x}
$$

The algebraic structure of the local symmetries induces a co-product $\Delta$, a counit $\epsilon$, an antipode $\mathcal{S}$ and a $R$-matrix for the full gauge symmetry $\mathcal{G}$ such that $\mathcal{G}$ becomes a quasitriangular Hopf-*-algebra in the sense discussed above. Ribbon elements $v_{x} \in \mathcal{G}_{x}$ furnish a ribbon element $v$ for $\mathcal{G}$.

\section{$3.2 \quad$ Representation theory of $\mathcal{G}$}

We start a discussion of the representation theory of $\mathcal{G}$ with some general remarks. Given two representations $\tau, \tau^{\prime}$ of a Hopf-algebra $\mathcal{G}$, their tensor product $\tau \otimes \tau^{\prime}$ is defined with the help of the co-product $\Delta$

$$
\left(\tau \otimes \tau^{\prime}\right)(\xi) \equiv\left(\tau \otimes \tau^{\prime}\right)(\Delta(\xi)) .
$$

The co-unit $\epsilon$ is a one-dimensional representation of $\mathcal{G}$. It is a trivial representation in the sense that $(\epsilon \otimes \tau)(\xi)=\tau(\xi)=(\tau \otimes \epsilon)(\xi)$ holds for all $\xi \in \mathcal{G}$. A representation $\tau$ on a Hilbert space $V$ is called unitary, if $\tau\left(\xi^{*}\right)=\tau(\xi)^{*}$ for all $\xi \in \mathcal{G}$. Note that the tensor product of two unitary representations $\tau, \tau^{\prime}$ is not 
unitary in general (provided that we use the standard scalar product on the tensor product of Hilbert spaces). Instead we have

$$
\left(\tau \otimes \tau^{\prime}\right)\left(\Delta^{\prime}\left(\xi^{*}\right)\right)=\left(\left(\tau \otimes \tau^{\prime}\right)(\Delta(\xi))^{*} .\right.
$$

The (nonunitary) matrix $\left(\tau \otimes \tau^{\prime}\right)(R)$ furnishes an intertwiner between the representations $\left(\tau \otimes \tau^{\prime}\right) \circ \Delta^{\prime}$ and $\left(\tau \otimes \tau^{\prime}\right) \circ \Delta$.

There are two natural "contragradient" representations which come with the antipode $\mathcal{S}$. They are obtained as

$$
\begin{aligned}
& \tilde{\tau}(\xi) \equiv{ }^{t} \tau\left(\mathcal{S}^{-1}(\xi)\right), \\
& \bar{\tau}(\xi) \equiv{ }^{t} \tau(\mathcal{S}(\xi)),
\end{aligned}
$$

for all $\xi \in \mathcal{G}$. Here ${ }^{t}$ denotes the transpose of matrices. The relations (3.3) assert that $\tilde{\tau}$ and $\bar{\tau}$ are equivalent but non-equal unless $u=e$. Unitarity of $\tau$ results in the unitarity of both contragradient representations. The tensor products $\tau \otimes \bar{\tau}, \bar{\tau} \otimes \tau$ contain $\epsilon$ as a subrepresentation (hence the name "contragradient"). These properties can be abstracted from the relations

$$
\sum_{\sigma} \bar{\tau}_{a b}\left(\xi_{\sigma}^{1}\right) \tau_{a c}\left(\xi_{\sigma}^{2}\right)=\epsilon(\xi) \delta_{b, c}, \sum_{\sigma} \tau_{c b}\left(\xi_{\sigma}^{1}\right) \bar{\tau}_{a b}\left(\xi_{\sigma}^{2}\right)=\epsilon(\xi) \delta_{a, c},
$$

which are a direct consequence of the definition of the antipode $\mathcal{S}$. Here $\xi_{\sigma}^{i}$ are defined via the decomposition of the coproduct:

$$
\Delta(\xi)=\sum_{\sigma} \xi_{\sigma}^{1} \otimes \xi_{\sigma}^{2}
$$

Representations of the algebra $\mathcal{G}$ of gauge transformations are obtained as families $\left(\tau_{x}\right)_{x \in S}$ of representations of the symmetries $\mathcal{G}_{x}$. We are mainly interested in those representations of $\mathcal{G}$ which come from the same representation of $\mathcal{G}_{*}$. At this point let us assume that $\mathcal{G}_{*}$ is semisimple and that every equivalence class $[J]$ of irreducible representations of $\mathcal{G}_{*}$ contains a unitary representative $\tau_{*}^{J}$ with carrier space $V^{J}$. For the moment, the most interesting examples of gauge symmetries - e.g. $U_{q}\left(s l_{2}\right), q^{p}=1$ - are ruled out by this assumption. This will be revisited in section 7 . Tensor products $\tau_{*}^{I} \otimes \tau_{*}^{J}$ can be decomposed into irreducibles $\tau_{*}^{K}$. This decomposition determines the Clebsch-Gordon maps $C_{*}^{a}[I J \mid K]: V^{I} \otimes V^{J} \mapsto V^{K}$,

$$
C_{*}^{a}[I J \mid K]\left(\tau_{*}^{I} \otimes \tau_{*}^{J}\right)(\xi)=\tau_{*}^{K}(\xi) C_{*}^{a}[I J \mid K] .
$$

The same representations $\tau_{*}^{K}$ in general appears with some multiplicity $N_{K}^{I J}$. The superscript $a=1, \ldots, N_{K}^{I J}$ keeps track of these subrepresentations. It is common to call the numbers $N_{K}^{I J}$ fusion rules. Normalization of Clebsch Gordon maps is connected with an extra assumption. It will be central for the positivity later. Notice that the ribbon element $v_{*}$ is central so that the evaluation with 
irreducible representations $\tau_{*}^{I}$ gives complex numbers $v^{I}=\tau_{*}^{I}\left(v_{*}\right)$. We suppose that there exists a set of square roots $\kappa_{I}, \kappa_{I}^{2}=v^{I}$, such that

$$
C_{*}^{a}[I J \mid K]\left(\tau_{*}^{I} \otimes \tau_{*}^{J}\right)\left(R_{*}^{\prime}\right) C_{*}^{b}[I J \mid L]^{*}=\delta_{a, b} \delta_{K, L} \frac{\kappa_{I} \kappa_{J}}{\kappa_{K}} .
$$

Here $R_{*}^{\prime}=\sum r_{* \sigma}^{2} \otimes r_{* \sigma}^{1}$. Let us analyze this relation in more detail. As a consequence of intertwining properties of the Clebsch Gordon maps and the $R$ element, $\tau_{*}^{K}(\xi)$ commutes with the left hand side of the equation. So by Schurs' lemma, it is equal to the identity $e^{K}$ times some complex factor $\omega_{a b}(I J \mid K)$. After appropriate normalization, $\omega_{a b}(I J \mid K)=\delta_{a, b} \omega(I J \mid K)$ with a complex phase $\omega(I J \mid K)$. Next we exploit the $*$-operation and relation (3.6) to find $\omega_{a b}(I J \mid K)^{2}=v^{I} v^{J} / v^{K}$. This means that (3.13) can be ensured up to a possible $\operatorname{sign} \pm$. Here we assume that this sign is always + . This assumption is met by the quantized universal enveloping algebras of all simple Lie algebras because they are obtained as a deformation of a Hopf-algebra which clearly satisfies (3.13).

We wish to combine the phases $\kappa_{I}$ into one element $\kappa_{*}$ in the center of $\mathcal{G}_{*}$, i.e. by definition, $\kappa_{*}$ will denote a central element

$$
\kappa_{*} \in \mathcal{G}_{*} \quad \text { with } \quad \tau_{*}^{J}\left(\kappa_{*}\right)=\kappa_{J} .
$$

Such an element does exist and is unique. It has the property $\kappa_{*}^{*}=\kappa_{*}^{-1}$.

The antipode $\mathcal{S}_{*}$ furnishes a conjugation in the set of equivalence classes of irreducible representations. We use $[\bar{J}]$ to denote the class conjugate to $[J]$. It is defined such that the two equivalent contragradient representations $\tilde{\tau}_{*}^{J} \bar{\tau}_{*}^{J}$ of the representative $\tau_{*}^{J} \in[J]$ are elements in the conjugate class $[\bar{J}]$.

Let us finally mention that the trace of the element $\mathcal{S}_{*}\left(u_{*}\right) v_{*}^{-1}$ in a given representation $\tau^{J}$ computes the "quantum dimension" $d_{J}$ of the representation $\tau^{J}[28]$, i.e.

$$
d_{J}=\operatorname{dim}_{q}\left(V^{J}\right) \equiv \operatorname{Tr}\left(\tau^{J}\left(\mathcal{S}_{*}\left(u_{*}\right) v_{*}^{-1}\right)\right) .
$$

Representations and intertwiners of $\mathcal{G}_{*}$ are now transported to the algebras $\mathcal{G}_{x}$. This is accomplished with the help of isomorphisms $\iota_{x}$ and twist elements $F_{x}$.

$$
\begin{aligned}
\tau_{x}^{I}(\xi) & =\tau_{*}^{I}\left(\iota_{x}(\xi)\right) \text { for all } \xi \in \mathcal{G}_{x}, \\
C_{x}^{a}[I J \mid K] & =C_{*}^{a}[I J \mid K]\left(\tau_{*}^{I} \otimes \tau_{*}^{J}\right)\left(F_{x}^{-1}\right) .
\end{aligned}
$$

The representations $\tau_{x}^{I}$ act on the space $V_{x}^{I}=V^{I}$. It is immediately checked that the new maps $C_{x}$ satisfy the standard intertwining relations

$$
\tau_{x}^{K}(\xi) C_{x}^{a}[I J \mid K]=C_{x}^{a}[I J \mid K]\left(\tau_{x}^{I} \otimes \tau_{x}^{J}\right)\left(\Delta_{x}(\xi)\right) \text { for all } \xi \in \mathcal{G}_{x} .
$$

Similar relations hold for the adjoint intertwiners

$$
\begin{aligned}
C_{x}^{a}[I J \mid K]^{*} & =\left(\tau_{*}^{I} \otimes \tau_{*}^{J}\right)\left(F_{x}^{\prime}\right) C_{*}^{a}[I J \mid K]^{*}, \\
C_{x}^{a}[I J \mid K]^{*} \tau_{x}^{K}(\xi) & =\left(\tau_{x}^{I} \otimes \tau_{x}^{J}\right)\left(\Delta_{x}^{\prime}(\xi)\right) C_{x}^{a}[I J \mid K]^{*} \text { for all } \xi \in \mathcal{G}_{x} .
\end{aligned}
$$


Finally, the central element $\kappa_{*} \in \mathcal{G}_{*}$ introduced in eq. (3.14) is transported to central elements in $\mathcal{G}_{x}$ with the help of the formula $\kappa_{x}=\iota_{x}^{-1}\left(\kappa_{*}\right)$.

So far we have only described the representation theory of the $\mathcal{G}_{x}$. Among all the representations of the total algebra $\mathcal{G}$ of gauge transformations which can be built from representations of the $\mathcal{G}_{x}$, we need only one family $\left(\tau^{I, i}\right)_{i \in L}$ assigned to the links of the graph. The representations $\tau^{I, i}$ of $\mathcal{G}$ will later describe the transformation properties of the basic quantum variables $U^{I}(i)$, i.e. of the parallel transporters along the link $i$.

$$
\tau^{I, i}(\xi) \equiv\left\{\begin{array}{cl}
\tau_{y}^{I}(\xi) & \text { if } \xi \in \mathcal{G}_{y} \\
\bar{\tau}_{x}^{I}(\xi) & \text { if } \xi \in \mathcal{G}_{x} \\
\epsilon_{z}(\xi) & \text { else }
\end{array}\right.
$$

for $x=t(-i), y=t(i)$. To decompose tensor products of representations $\tau^{I, i}$, $\tau^{J, i}$ assigned to the same link $i$, we use the following intertwiners

$$
C^{a}[I J \mid K]^{i} \equiv C_{y}^{a}[I J \mid K] \otimes{ }^{t}\left(C_{x}^{a}[I J \mid K]^{*}\right) .
$$

As usual, ${ }^{t}$ denotes the transpose. $C^{a}[I J \mid K]^{i}$ is a map from $\left(V_{y}^{I} \otimes V_{y}^{J}\right) \otimes\left(V_{x}^{I} \otimes\right.$ $\left.V_{x}^{J}\right)$ to $V_{y}^{K} \otimes V_{x}^{K}$ which enjoys the intertwining property

$$
\tau^{K, i}(\xi) C^{a}[I J \mid K]^{i}=C^{a}[I J \mid K]^{i}\left(\tau^{I, i} \otimes \tau^{J, i}\right)(\Delta(\xi)) \quad \text { for all } \quad \xi \in \mathcal{G} .
$$

There are further relations between representations on the same link, which involve both orientations $i,-i$. In fact, there is an equivalence between the representations $\tau^{I, i}$ and $\tilde{\tau}^{I,-i}$. Let us describe this explicitly. By rel. (3.3), the element $\mathcal{S}_{y}\left(u_{y}\right)$ furnishes intertwiners $\eta_{y}^{I}=\tau_{y}^{I}\left(\mathcal{S}_{y}\left(u_{y}\right)\right)$ with the property

$$
\eta_{y}^{I} \tau_{y}^{I}\left(\mathcal{S}_{y}(\xi)\right)=\tau_{y}^{I}\left(\mathcal{S}_{y}^{-1}(\xi)\right) \eta_{y}^{I}
$$

From this equation one deduces that

$$
\begin{aligned}
\eta^{I, i} \tilde{\tau}^{I, i}(\xi) & =\tau^{I,-i}(\xi) \eta^{I, i} \\
\text { with } \eta^{I, i} & \equiv e_{x}^{I} \otimes{ }^{t} \eta_{y}^{I}
\end{aligned}
$$

Here $e_{x}^{J}$ is the identity on $V_{x}^{I}=V^{I}$. A similar equivalence appears between the representations $\tau^{I,-i}$ and $\tau^{\bar{I}, i}$. This time the intertwiner is constructed from the Clebsch Gordon maps. We introduce it according to

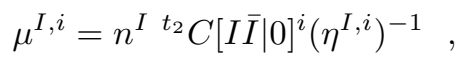

where ${ }^{t_{2}}$ means transpose only with respect to the second component and $n^{I}=n^{\bar{I}}$ is a normalization determined by $\mu^{I, i} \mu^{\bar{I},-i}=i d$. The element $\mu^{I, i}$ enjoys the intertwining property

$$
\mu^{I, i} \tau^{I,-i}(\xi)=\tau^{\bar{I}, i}(\xi) \mu^{I, i}
$$




\section{Quantum group valued gauge fields}

In this section we plan to introduce our basic lattice algebra $\mathcal{B}$. It is an algebra generated by the quantum lattice connections $U^{I}$ together with the quantum gauge transformations $\xi \in \mathcal{G}$ discussed in the preceding section. Relations between the elements $U^{I}$ and the gauge transformations $\xi \in \mathcal{G}$ are determined by the covariance properties of the quantum lattice connection. All other relations among elements $U^{I}(i)$ are postulated in the spirit of section 2 .

\subsection{Definition of the lattice algebra $\mathcal{B}$}

To define the lattice algebra $\mathcal{B}$ we have to introduce some extra structure on the graph $G$. The orientation of the Riemann surface $\Sigma$ determines a canonical cyclic order in the set $L_{x}=\{i \in L: t(i)=x\}$ of links incident to the vertex $x$. Writing the relations in $\mathcal{B}$ we are forced to specify a linear order within $L_{x}$. To this end one considers ciliated graphs $G_{c i l}$. A ciliated graph can be represented by picturing the underlying graph together with a small cilium $c_{x}$ at each vertex. For $i, j \in L_{x}$ we write $i \leq j$, if $\left(c_{x}, i, j\right)$ appear in a clockwise order.

Definition 1 (Lattice algebra $\mathcal{B})$ The associative algebra $\mathcal{B}=\mathcal{B}\left(G_{\text {cil }}\right)$ is generated by elements $U_{\alpha}^{I}(i)=U_{a_{1} a_{2}}^{I}(i), i \in L, \alpha=1, \ldots, \operatorname{dim}\left(\tau^{I, i}\right)$, and the elements of $\mathcal{G}$ such that

1. the unit element $e$ of $\mathcal{G}$ acts as a unit element of $\mathcal{B}$, i.e. $U_{\alpha}^{I}(i) e=U_{\alpha}^{I}(i)=$ $e U_{\alpha}^{I}(i)$.

2. the tuples $\left(U_{\alpha}^{I}(i)\right)$ transform covariantly according to the representation $\tau^{I, i}$

$$
\xi U_{\alpha}^{I}(i)=U_{\beta}^{I}(i)\left(\tau_{\beta \alpha}^{I, i} \otimes i d\right)(\Delta(\xi)) \quad \text { for all } \quad \xi \in \mathcal{G} .
$$

3. "functoriality" holds on the links

$$
\begin{array}{r}
U_{\alpha}^{I}(i) U_{\beta}^{J}(i)=\sum U_{\gamma}^{K}(i) C^{a}\left[\begin{array}{ccc}
I & J & K \\
\alpha & \gamma
\end{array}\right]^{i}, \\
U_{a b}^{I}(i) U_{c b}^{I}(-i)=\delta_{a, c} \quad, \quad U_{b a}^{I}(-i) U_{b c}^{I}(i)=\delta_{a, c} .
\end{array}
$$

Here C[..] are matrix elements of the Clebsch Gordon intertwiners (3.10) introduced in the last section.

4. elements $U_{\alpha}^{I}(i)$ satisfy braid relations

$$
U_{\alpha}^{I}(i) U_{\beta}^{J}(j)=U_{\gamma}^{J}(j) U_{\delta}^{I}(i)\left(\tau_{\delta \alpha}^{I, i} \otimes \tau_{\gamma \beta}^{J, j}\right)(R) .
$$

for $i \leq j$ or if $i, j$ have no common endpoints. 
This definition is rather central and requires some thoughtful discussion. Intuitively, we prefer to think about the generators $U_{a_{1} a_{2}}^{I}(i)$ as elements of a matrix. Nevertheless proofs often simplify if we regard them as vectors in a $\operatorname{dim}\left(\tau^{I, i}\right)$-dimensional vector space. Whenever we adopt the second point of view, we use the multiindex $\alpha$ instead of its components $a_{1}, a_{2}$.

The covariance relation in 2 . can be written in a more explicit form if we insert the expansion $\Delta(\xi)=\sum \xi_{\sigma}^{1} \otimes \xi_{\sigma}^{2}$.

$$
\xi U_{\alpha}^{I}(i)=U_{\beta}^{I}(i) \tau_{\beta \alpha}^{I, i}\left(\xi_{\sigma}^{1}\right) \xi_{\sigma}^{2}
$$

This tells us how to shift elements $\xi \in \mathcal{G}$ through factors $U_{\alpha}^{I}(i)$ from left to right. We note a simple consequence of this fact.

Proposition 2 Every element of $\mathcal{B}$ is a complex linear combination of elements of the form

$$
U_{\alpha_{1}}^{I_{1}}\left(i_{1}\right) \ldots U_{\alpha_{n}}^{I_{n}}\left(i_{n}\right) \xi \quad \text { with } \quad n \geq 0, \xi \in \mathcal{G}
$$

The relations (4.1) appear as a special case of a more general notion of covariance.

Definition 3 ( $\mathcal{G}$ (right-) covariance) Let $\tau=\left(\tau_{\alpha \beta}\right)_{\alpha, \beta \in I}$ be a representation matrix of a n-dimensional representation of $\mathcal{G}$. An n-tuple $F=\left(F_{\alpha}\right)_{\alpha \in I}, F_{\alpha} \in \mathcal{B}$, is said to transform (right-) covariantly according to the representation $\tau$ of $\mathcal{G}$ if

$$
\xi F_{\alpha}=F_{\beta}\left(\tau_{\beta \alpha} \otimes i d\right)(\Delta(\xi))
$$

for all $\xi \in \mathcal{G} . F \in \mathcal{B}$ is called $\mathcal{G}$-invariant if it transforms according to the trivial representation $\epsilon$ of $\mathcal{G}$, or equivalently, if

$$
\xi F=F \xi
$$

for all $\xi \in \mathcal{G}$.

Indeed this is an appropriate notion of covariance. Assume for a moment that $\xi$ is an element of a unitary group rather than a general Hopf algebra. Then the co-product and the $*$-operation act according to $\Delta(\xi)=\xi \otimes \xi$ and $\xi^{*}=\xi^{-1}$. So the covariance relation (4.5) simplifies to $\xi F_{\alpha} \xi^{*}=F_{\beta} \tau_{\beta \alpha}(\xi)$.

After this preparation we see that the covariance (4.1) can be regarded as a quantum version of the classical relation 2.29). The latter means that the variable $U_{a_{1} a_{2}}^{I}(i)$ transforms covariantly according to the representation $\tau_{y}^{I}$ in the second index while it transforms according to the representation ${ }^{t} \tau_{x}^{I} \circ \mathcal{S}_{x}$ in the first index (if $i$ points from $x$ to $y$ ). This is encoded in the definition of $\tau^{I, i}$.

We will often have to move elements $\xi \in G$ from right to left. According to the following proposition, this is always possible. 
Proposition 4 (left covariance) Suppose that the tuple $\left(F_{\alpha}\right), F_{\alpha} \in \mathcal{B}$ transforms covariantly according to the representation $\tau$ of $\mathcal{G}$. Then we have

$$
F_{\alpha} \xi=\left(\tilde{\tau}_{\alpha \beta} \otimes i d\right)(\Delta(\xi)) F_{\beta}
$$

for all $\xi \in \mathcal{G}$. In other words, every right-covariant tuple in $\mathcal{B}$ is also leftcovariant.

Proof: We write the covariance relation for the components $\xi_{\sigma}^{2}$ in the expansion of $\Delta(\xi)$.

$$
\xi_{\sigma}^{2} F_{\alpha}=F_{\beta} \tau_{\beta \alpha}\left(\xi_{\sigma \tau}^{21}\right) \xi_{\sigma \tau}^{22}
$$

Multiplication with $\tau_{\alpha \gamma}\left(\mathcal{S}^{-1}\left(\xi_{\sigma}^{1}\right)\right)$, summation over $\sigma$ and the co-associativity of $\Delta$ lead to

$$
\begin{aligned}
\xi_{\sigma}^{2} F_{\alpha} \tau_{\alpha \gamma}\left(\mathcal{S}^{-1}\left(\xi_{\sigma}^{1}\right)\right) & =F_{\beta} \tau_{\beta \gamma}\left(\xi_{\sigma \tau}^{12} \mathcal{S}^{-1}\left(\xi_{\sigma \tau}^{11}\right)\right) \xi_{\sigma}^{2} \\
& =F_{\gamma} \epsilon\left(\xi_{\sigma}^{1}\right) \xi_{\sigma}^{2}=F_{\gamma} \xi
\end{aligned}
$$

The left hand side of this equation is equal to $\tilde{\tau}_{\gamma \alpha}\left(\xi_{\sigma}^{1}\right) \xi_{\sigma}^{2} F_{\alpha}$.

This concludes our discussion of item 2. above. Let us turn to functoriality next. At the end of the preceding section we described a number of equivalences between representations assigned to the link i. The relations in 3. mean that all these equivalences reflect themselves as equalities among the variables $U_{\alpha}^{I}(i)$. While this explains the term "functoriality" it is much more instructive to check that the postulated relations are consistent with the covariance. This is done by comparison of the definitions in 2., 3. with the intertwining relations (3.17) of $C^{a}[I J \mid K]^{i}$ and property (3.10).

Equations (4.2) should be regarded as a kind of operator product expansions. They can be written in a form which comes close to the classical relations (2.52), if the definition (3.16) of $C^{a}[I J \mid K]^{i}$ is inserted. The set of relations (4.3) reflect the behaviour of $U^{I}(i)$ under $i \rightarrow-i$. In the formulation given in 3. they look exactly like their counterparts (2.43) in section 2 . In the quantum algebra $\mathcal{B}$ we would like to substitute (4.3) by a new set of relations which is manifestly covariant. Using the operator product expansions (4.2) one derives

$$
U_{\alpha}^{I}(-i)=U_{\beta}^{\bar{I}}(i) \mu_{\beta \alpha}^{I, i} .
$$

(The element $\mu^{I, i}$ was defined in (3.19)). In fact, relations (4.2,4.8) are equivalent to the pair 4.2,4.3) and thus furnish a new definition of $\mathcal{B}$. The latter implies that every product of elements $U_{\alpha}^{I}(i)$ and $U_{\beta}^{I}(-i)$ is a complex linear combination of $U_{\beta}^{J}(i)$.

We can now proceed to the discussion of item 4.. Of course braid relations substitute for the commutation relations of classical lattice connections. The braid relations between the components of $U^{I}(i), U^{J}(j), i \leq j$, are almost 
uniquely determined by the consistency with the transformation law and with the associativity of the product in $\mathcal{B}$. Since $\left(\tau^{I, i} \otimes \tau^{J, j}\right)(R)$ furnishes an intertwiner between the representations $\tau^{I, i} \otimes \tau^{J, j}$ and $\tau^{J, j} \otimes \tau^{I, i}$, both sides of the braid relations transform according to the same representation $\tau^{I, i} \otimes \tau^{J, j}$. Consistency with the associativity relies on the Yang Baxter equation for $R$. One should also notice that these braid relations require the introduction of eyelashes.

Actually the braid relations in the definition of $\mathcal{B}$ are identical to the corresponding relations in section 2. If $i, j$ have no common endpoints then $\left(\tau^{I, i} \otimes\right.$ $\left.\tau^{J, j}\right)(R)$ is the identity matrix so that the corresponding variables $U^{I}(i), U^{J}(j)$ commute. Suppose next that the links $i, j$ point towards the same vertex $x$ while their second endpoints are disjoint. Then $\left(\tau^{I, i} \otimes \tau^{J, j}\right)(R)=\left(\tau_{y}^{I} \otimes \tau_{y}^{J}\right)\left(R_{y}\right)$ and this matrix acts only on the second component of the indices $\gamma=\left(c_{1}, c_{2}\right), \delta=$ $\left(d_{1}, d_{2}\right)$. So we end up with the relations (2.47) if $i<j$. Finally we come to the case $i=j$, where the $R$-matrix in 4 . picks up contributions from both endpoints of the link $i$. More precisely $\left(\tau^{I, i} \otimes \tau^{J, j}\right)(R)$ is equal to the matrix $\left(\tau_{y}^{I} \otimes \tau_{y}^{J}\right)\left(R_{y}\right)\left({ }^{t} \tau_{x}^{I} \otimes{ }^{t} \tau_{x}^{J}\right)\left(R_{x}\right)$ acting on both components of the indices $\gamma, \delta$. To see this one uses that $\left(\mathcal{S}_{x} \otimes \mathcal{S}_{x}\right)\left(R_{x}\right)=R_{x}$. Consequently, the braid relations (4.4) can be written in the form of relation (2.46).

The braid relations spelled out in 4 . do not determine the commutation relations for arbitrary choice of the links $i, j$. For example if $i,-j$ point towards the same endpoint $x$, the commutation relations for $U^{I}(i), U^{J}(j)$ are not stated explicitly. However they can be derived from the relations among $U^{I}(i), U^{\bar{J}}(-j)$. The reason is that (4.8) provides a complex linear relation between $U^{I}(i)$ and $U^{\bar{I}}(-i)$. As an example we give the relations for $-i,-j$ if $i \leq j$.

$$
U_{\alpha}^{I}(-i) U_{\beta}^{J}(-j)=\left(\tilde{\tau}_{\beta \delta}^{J,-j} \otimes \tilde{\tau}_{\alpha \delta}^{I,-i}\right)\left(R^{\prime}\right) U_{\delta}^{J}(-j) U_{\gamma}^{I}(-i)
$$

The reader is invited to verify this relation explicitly. We arrive at the rather important conclusion that any two variables $U^{I}(i), U^{J}(j)$ can be (braid-) commuted.

Proposition 5 Suppose that $i_{1}, i_{2}, \ldots i_{n}$ is a maximal ordered set of oriented links with the property that every link appears only once and only in one orientation, i.e. $\pm i_{\nu} \neq i_{\mu}$ for all $\nu \neq \mu$. Then any element of $\mathcal{B}$ is a complex linear combination of elements

$$
U_{\alpha_{1}}^{I_{1}}\left(i_{1}\right) \ldots U_{\alpha_{n}}^{I_{n}}\left(i_{n}\right) \xi \quad \text { with } n \geq 0, \xi \in \mathcal{G} .
$$

The following proposition asserts that the functoriality is consistent with the braid relations.

Proposition 6 (braid relations for composite operators) Suppose that $F=$ $\left(F_{\alpha}\right), F^{\prime}=\left(F_{\beta}^{\prime}\right)$ and $F^{\prime \prime}=\left(F_{\gamma}^{\prime \prime}\right)$ transform covariantly according to representations $\tau, \tau^{\prime}$ and $\tau^{\prime \prime}$ of $\mathcal{G}$. 
(i) Suppose that the braid relations

$$
\begin{aligned}
& F_{\alpha} F_{\beta}^{\prime}=F_{\mu}^{\prime} F_{\nu}\left(\tau_{\nu \alpha} \otimes \tau_{\mu \beta}^{\prime}\right)(R), \\
& F_{\alpha} F_{\gamma}^{\prime \prime}=F_{\mu}^{\prime \prime} F_{\rho}\left(\tau_{\rho \alpha} \otimes \tau_{\mu \beta}^{\prime \prime}\right)(R),
\end{aligned}
$$

hold true. Then $F$ and $F^{\prime} F^{\prime \prime}$ satisfy braid relations

$$
F_{\alpha} F_{\beta}^{\prime} F_{\gamma}^{\prime \prime}=F_{\mu}^{\prime} F_{\nu}^{\prime \prime} F_{\rho}\left(\tau_{\rho \alpha} \otimes\left(\tau^{\prime} \otimes \tau^{\prime \prime}\right)_{\mu \nu, \beta \gamma}\right)(R) .
$$

The proof of this proposition is a standard application of the quasi-triangularity relation of $R$ (cp. [1] for details).

Before we finish our discussion on definition 11 we want to remark that an algebra similar to $\mathcal{B}$ was proposed by Boulatov 5 . In his approach, variables assigned to different links commute. We see that this is in general not consistent with the local quantum symmetry of the model, i.e. by the consistency with the transformation law under local quantum symmetry transformations one is forced to use braid relations instead of ordinary commutation.

\subsection{The *-operation on $\mathcal{B}$}

We will obtain the observables of Chern Simons as a subalgebra of $\mathcal{B}$. In quantum physics, observables come with a ${ }^{*}$-operation. This *-operation will be a reminiscent of a ${ }^{*}$-operation in $\mathcal{B}$. The construction of the latter is the main topic within this subsection.

Proposition 7 (anti-homomorphism $\theta$ ) There is a unique anti-homomorphism $\theta: \mathcal{B} \mapsto \mathcal{B}$ with the properties

$$
\begin{aligned}
\theta(\xi) & =\xi^{*} \\
\theta\left(U_{\alpha}^{I}(i)\right) & =U_{\gamma}^{I}(-i)\left(\tau_{\gamma \beta}^{I,-i} \otimes i d\right)\left(R^{-1}\right) \eta_{\beta \alpha}^{I, i} .
\end{aligned}
$$

In particular, $\theta$ extends the $*$-operation on $\mathcal{G} \subset \mathcal{B}$.

"Conjugations" of this type were first proposed in [2] (cp. also [9] for a simple example). If the $R$-matrix would be trivial (as it is for group algebras), the action of $\theta$ would simplify to $\theta\left(U_{\alpha}^{I}(i)\right)=U_{\alpha}^{I}(-i)$. This is the familiar unitarity of the lattice connection. The formula (4.13) looks more convincing, if we use the elements $R_{x}, R_{y}$ instead of $R$. One can check that

$$
\theta\left(U_{a d}^{I}(i)\right)=\left(\tau_{x}^{I} \otimes i d\right)_{a b}\left(R_{x}\right) U_{b c}^{I}(-i)\left(\tau_{y}^{I} \otimes i d\right)_{c d}\left(R_{y}^{-1}\right) .
$$

We start the proof of the proposition with the following lemma.

Lemma 8 With $v_{I}=\tau^{I}\left(v_{*}\right)$ the expression (4.13) for $\theta$ can be rewritten according to

$$
\theta\left(U_{\alpha}^{I}(i)\right)=v_{I}^{-2} \eta_{\alpha \beta}^{I,-i}\left(\tilde{\tau}_{\gamma \beta}^{I,-i} \otimes i d\right)(R) U_{\gamma}^{I}(-i)
$$


Proof: To prove this relation we apply the covariance relation (proposition (1) to move the $R$-matrix from the right to the left and insert the definition of $\tilde{\tilde{\tau}}^{I,-i}$.

$$
\begin{aligned}
\theta\left(U_{\alpha}^{I}(i)\right) & =\left(\tau_{\gamma \beta}^{I,-i} \otimes \tilde{\tau}_{\gamma \delta}^{I,-i} \otimes i d\right)\left((i d \otimes \Delta)\left(R^{-1}\right)\right) U_{\delta}^{I}(-i) \eta_{\beta \alpha}^{I, i} \\
& =\left(\tilde{\tau}_{\beta \gamma}^{I,-i} \otimes \tilde{\tau}_{\gamma \delta}^{I,-i} \otimes i d\right)\left((\mathcal{S} \otimes i d \otimes i d)(i d \otimes \Delta)\left(R^{-1}\right)\right) U_{\delta}^{I}(-i) \eta_{\beta \alpha}^{I, i} \\
& =\left(\tilde{\tau}_{\beta \delta}^{I,-i} \otimes i d\right)\left((\mathcal{S} \otimes i d) R^{-1}\left(\mathcal{S}\left(u^{-1}\right) \otimes e\right)\right) U_{\delta}^{I}(-i) \eta_{\beta \alpha}^{I, i} .
\end{aligned}
$$

The last step uses the quasi-triangularity of $R$ and the definition of $u(3.3)$. Now $(\mathcal{S} \otimes i d) R^{-1}\left(\mathcal{S}\left(u^{-1}\right) \otimes e\right)=\left(\mathcal{S}\left(u^{-1}\right) \otimes e\right)\left(\mathcal{S}^{-1} \otimes i d\right) R^{-1}=\left(\mathcal{S}\left(u^{-1}\right) \otimes e\right) R$ and with the definition (3.18) of $\eta^{I, i}$ this finally gives the formula anticipated in the lemma.

Proof of proposition 7 : Since the action of $\theta$ is specified on all generators of $\mathcal{B}$, uniqueness is obvious. We have to show that the extension of $\theta$ to $\mathcal{B}$ is consistent with the relations in $\mathcal{B}$. The simplest part is the consistency with the covariance relations. We apply $\theta$ to the right hand side of the covariance relation (definition 1.2) and use a series of intertwining relations.

$$
\begin{aligned}
& \theta\left(U_{\beta}^{I}(i)\left(\tau_{\beta \alpha}^{I, i} \otimes i d\right) \Delta(\xi)\right) \\
= & v_{I}^{-2}\left(\tau_{\alpha \beta}^{I, i} \otimes i d\right) \Delta^{\prime}\left(\xi^{*}\right) \eta_{\beta \gamma}^{I,-i}\left(\tilde{\tau}_{\gamma \delta}^{I,-i} \otimes i d\right)(R) U_{\delta}^{I}(-i) \\
= & v_{I}^{-2} \eta_{\alpha \beta}^{I,-i}\left(\tilde{\tau}_{\beta \gamma}^{I,-i} \otimes i d\right)(R)\left(\tilde{\tau}_{\gamma \delta}^{I,-i} \otimes i d\right) \Delta\left(\xi^{*}\right) U_{\delta}^{I}(-i) \\
= & v_{I}^{-2} \eta_{\alpha \beta}^{I,-i}\left(\tilde{\tau}_{\beta \gamma}^{I,-i} \otimes i d\right)(R) U_{\gamma}^{I}(-i) \xi^{*}=\theta\left(\xi U_{a}^{I}(i)\right) .
\end{aligned}
$$

The reader is invited to check this calculation carefully. The consistency with the covariance relations provides the main motivation for the definition of $\theta$. The factor

$$
\left(\tau_{\gamma \beta}^{I,-i} \otimes i d\right)\left(R^{-1}\right) \eta_{\beta \alpha}^{I, i}
$$

which appears in $\theta\left(U_{\alpha}^{I}(i)\right)$ is designed to match the different transformation laws of $\theta\left(U_{\alpha}^{I}(i)\right)$ and $U_{\beta}^{I}(-i)$.

Let us turn to the braid relations next. If $i, j$ have no common endpoints, the relations which result after the applications of $\theta$ are obviously identical to the commutation relations for $U^{I}(-i), U^{J}(-j)$. So let us concentrate on the case $i \leq j$. We have to check that

$$
\theta\left(U_{\beta}^{J}(j)\right) \theta\left(U_{\alpha}^{I}(i)\right)=\left(\tau_{\alpha \delta}^{I, i} \otimes \tau_{\beta \gamma}^{J, j}\right)\left(R^{\prime-1}\right) \theta\left(U_{\delta}^{I}(i)\right) \theta\left(U_{\gamma}^{J}(j)\right) .
$$

To do this we insert the formula for $\theta(U)$ given in the lemma above. After dividing by $v_{I}^{-2} \eta^{I, i}$ we obtain

$$
\begin{aligned}
& \left(\tilde{\tau}_{\beta \mu}^{J,-j} \otimes i d\right)(R) U_{\mu}^{J}(-j)\left(\tilde{\tau}_{\alpha \nu}^{I,-i} \otimes i d\right)(R) U_{\nu}^{I}(-i) \\
= & \left(\tilde{\tau}_{\alpha \delta}^{I,-i} \otimes \tilde{\tau}_{\beta \gamma}^{J,-j}\right)\left(R^{\prime-1}\right)\left(\tilde{\tau}_{\delta \rho}^{I,-i} \otimes i d\right)(R) U_{\rho}^{I}(-i)\left(\tilde{\tau}_{\gamma \sigma}^{J,-j} \otimes i d\right)(R) U_{\sigma}^{J}(-j) .
\end{aligned}
$$


We apply the transformation law (proposition A) to move all factors involving $R$ to the left.

$$
\begin{aligned}
& \left(\tilde{\tau}_{\alpha \nu}^{I,-i} \otimes \tilde{\tau}_{\beta \mu}^{J,-j} \otimes i d\right)\left(R_{23}(i d \otimes \Delta) R\right) U_{\mu}^{J}(-j) U_{\nu}^{I}(-i) \\
= & \left(\tilde{\tau}_{\beta \sigma}^{J,-j} \otimes \tilde{\tau}_{\alpha \rho}^{I,-i} \otimes i d\right)\left(R_{12}^{-1} R_{23}(i d \otimes \Delta) R\right) U_{\rho}^{I}(-i) U_{\sigma}^{J}(-j) .
\end{aligned}
$$

The quasi-triangularity of $R$ helps to simplify the product of $R$ matrices. More precisely, we apply

$$
(i d \otimes \Delta)\left(R^{-1}\right) R_{23}^{-1} R_{12} R_{13} R_{23} R_{12}^{\prime}=R_{12}^{\prime}
$$

to end up with the formula 4.9 . Consistency of $\theta$ with the relations $4.2,4.8$ ) is left as an exercise.

One is tempted to guess that $\theta$ gives a ${ }^{*}$-operation on $\mathcal{B}$ but this is not quite true. In fact it turns out that $\theta \circ \theta$ is non-equal to the identity unless $R^{\prime}=R^{-1}$. This is the content of the following calculation.

$$
\begin{aligned}
\theta \circ \theta\left(U_{\alpha}^{I}(i)\right) & =\theta\left(U_{\gamma}^{I}(-i)\left(\tau_{\gamma \beta}^{I,-i} \otimes i d\right)\left(R^{-1}\right) \eta_{\beta \alpha}^{I, i}\right) \\
& =\eta_{\alpha \beta}^{I, i *}\left(\tau_{\beta \gamma}^{I,-i} \otimes i d\right)\left(R^{\prime}\right) \theta\left(U_{\gamma}^{I}(-i)\right) \\
& =\eta_{\alpha \beta}^{I, i *}\left(\tau_{\beta \gamma}^{I,-i} \otimes i d\right)\left(R^{\prime}\right) v_{I}^{-2} \eta_{\gamma \delta}^{I, i}\left(\tilde{\tau}_{\delta \nu}^{I, i} \otimes i d\right)(R) U_{\nu}(i) \\
& =v_{I}^{-2}\left(\tilde{\tau}_{\alpha \nu}^{I, i} \otimes i d\right)\left(R^{\prime} R\right) U_{\nu}^{I}(i) \\
& =v U_{\alpha}^{I}(i) v^{-1}
\end{aligned}
$$

The result gives a concrete idea how $\theta$ can be improved to obtain an involution. Recall that we have introduced central elements $\kappa_{x} \in \mathcal{G}_{x}$ such that $\tau_{x}^{J}\left(\kappa_{x}\right)=v_{J}$. The set of $\kappa_{x}, x \in S$, determines an element $\kappa \in \mathcal{G}$ having the properties

$$
\kappa^{2}=v \quad, \quad \kappa^{*}=\kappa^{-1} .
$$

Conjugation with $\kappa$ gives an automorphism of $\mathcal{B}$ which can be used together with $\theta$ to construct the desired ${ }^{*}$-operation on $\mathcal{B}$. To formulate the result we define $\sigma_{\kappa}: \mathcal{B} \mapsto \mathcal{B}$,

$$
\sigma_{\kappa}(B)=\kappa^{-1} B \kappa \quad \text { for all } \quad B \in \mathcal{B}
$$

Theorem 9 (*-operation on $\mathcal{B})$ The anti-automorphism $\sigma_{\kappa} \circ \theta: \mathcal{B} \mapsto \mathcal{B}$ determines $a{ }^{*}$-operation on $\mathcal{B}$, i.e. $\left(\sigma_{k} \circ \theta\right)^{2}=i d$. We will write $B^{*} \equiv\left(\sigma_{k} \circ \theta\right)(B)$ for all $B \in \mathcal{B}$.

\section{The ${ }^{*}$-algebra $\mathcal{A}$ of observables}

We now come to the central part of this paper. The algebra $\mathcal{A}$ of observables, i.e. invariant elements generated by the gauge field, will be constructed. The 
*-operation on $\mathcal{B}$ can be restricted to $\mathcal{A}$. Even though the gauge fields depend on the position of cilia, the $*$-algebra $\mathcal{A}$ is essentially independent and thus $\mathcal{A}\left(G_{\text {cil }}\right)=\mathcal{A}(G)$. To avoid confusion about the term "observables" we should stress that the observables of the Chern Simons theory are only obtained after imposing the additional flatness conditions. This will be discussed in a forthcoming publication. The true observable algebras of Chern Simons will be identified as factor-algebras of $\mathcal{A}$ and all statements we make about $\mathcal{A}$ in the following in particular about the *-structure and positivity - imply corresponding results for Chern Simons observables.

\subsection{The definition of $\mathcal{A}$}

The elements $U_{\alpha}^{I}(i), i \in L$ generate a subalgebra of $\mathcal{B}$. It will be denoted by $<U_{\alpha}^{I}(i)>$.

Definition 10 (algebra of observables) The algebra $\mathcal{A}$ of observables is the invariant subalgebra of $\left\langle U_{\alpha}^{I}(i)\right\rangle$, i.e.

$$
\mathcal{A} \equiv\left\{A \in<U_{\alpha}^{I}(i)>\subset \mathcal{B} \mid \xi A=A \xi \text { for all } \xi \in \mathcal{G}\right\}
$$

$\mathcal{A}$ is spanned by elements of the form

$$
C_{\alpha_{1} \ldots \alpha_{n}} U_{\alpha_{1}}^{I_{1}}\left(i_{1}\right) \ldots U_{\alpha_{n}}^{I_{n}}\left(i_{n}\right) \quad n \geq 0
$$

where $C$ is supposed to possess the following intertwining property

$$
C\left(\tilde{\tau}^{I_{1}, i_{1}} \bowtie \ldots \bowtie \tilde{\tau}^{I_{n}, i_{n}}\right)(\xi)=\epsilon(\xi) C \quad \text { for all } \xi \in \mathcal{G} .
$$

Before we state our first result in this subsection we want to introduce the following shorthand notation

$$
\begin{aligned}
\Delta^{(1)}=\Delta & , \quad A^{(1)}=A, \\
\Delta^{(n+1)} & =\left(i d^{n} \otimes \Delta\right)\left(\Delta^{(n)}\right) \quad \text { for all } n \geq 1, \\
A^{(n+1)} & =\left(i d \otimes \Delta^{(n)}\right)(A) A^{(n)} \quad \text { for all } n \geq 1 .
\end{aligned}
$$

Here $A$ is an arbitrary element in $\mathcal{G} \otimes \mathcal{G}$.

Theorem 11 (*-operation on $\mathcal{A})$ The ${ }^{*}$-operation $\sigma_{k} \circ \theta: \mathcal{B} \mapsto \mathcal{B}$ restricts to a *-operation on the algebra $\mathcal{A}$ of observables. $\mathcal{A}$.

Proof: We show that $\theta$ maps all elements of the form (5.1) to elements in

$$
\begin{aligned}
& \theta\left(C_{\alpha_{1} \ldots \alpha_{n}} U_{\alpha_{1}}^{I_{1}}\left(i_{1}\right) \ldots U_{\alpha_{n}}^{I_{n}}\left(i_{n}\right)\right) \\
= & \theta\left(U_{\alpha_{n}}^{I_{n}}\left(i_{n}\right)\right) \ldots \theta\left(U_{\alpha_{1}}^{I_{1}}\left(i_{1}\right)\right) \overline{C_{\alpha_{1} \ldots \alpha_{n}}} \\
= & U_{\gamma_{n}}^{I_{n}}\left(-i_{n}\right) \ldots U_{\gamma_{1}}^{I_{1}}\left(-i_{1}\right)\left(\tau_{\gamma_{n} \beta_{n}}^{I,-i_{n}} \otimes \ldots \tau_{\gamma_{1} \beta_{1}}^{I,-i_{1}} \otimes i d\right)\left(\left(R^{-1}\right)^{(n)}\right) C_{\beta_{1} \ldots \beta_{n}}^{\prime} .
\end{aligned}
$$


Here we used the definition (5.2) for $\left(R^{-1}\right)^{(n)}$. Factors $\eta^{I, i}$ have been absorbed in the complex coefficients $C_{\beta_{1} \ldots \beta_{n}}^{\prime}$. $C^{\prime}$ has again a "good" intertwining property.

$$
\left(\tau^{I_{n},-i_{n}} \otimes \ldots \otimes \tau^{I_{1},-i_{1}}\right)(\xi) C^{\prime}=C^{\prime} \epsilon(\xi) \quad \text { for all } \quad \xi \in \mathcal{G} .
$$

Since $\left(R^{-1}\right)^{(n)}$ has $n+1$ components and we apply representation $\tau^{I,-i}$ only to the first $\mathrm{n}$ components, the above linear combination still has coefficients in $\mathcal{G}$. However one can show (e.g. by drawing a picture),

$$
\begin{aligned}
& \left(\tau_{\gamma_{n}, \beta_{n}}^{I_{n}, i_{n}} \otimes \ldots \tau_{\gamma_{1} \beta_{1}}^{I_{1},-i_{1}} \otimes i d\right)\left(\left(R^{-1}\right)^{(n)}\right) C_{\beta_{1} \ldots \beta_{n}}^{\prime} \\
= & \left(\tau_{\gamma_{n} \beta_{n}}^{I_{n},-i_{n}} \otimes \ldots \tau_{\gamma_{1} \beta_{1}}^{I_{1}, i_{1}}\right)\left(\left(R^{-1}\right)^{(n-1)}\right) C_{\beta_{1} \ldots \beta_{n}}^{\prime},
\end{aligned}
$$

so that the image of elements of the form (5.1) under $\theta$ is indeed an invariant element generated by the $U(i)^{\prime} s$. Since $\kappa$ is in $\mathcal{G}$, it commutes with all invariant elements in $\mathcal{B}$ and in particular with all the observables. Hence $\sigma_{k}(A)=A$ for all $A \in \mathcal{A}$ to that the assertion about $\sigma_{k} \circ \theta$ follows from what we said about $\theta$.

\subsection{Independence of the eyelash}

The braid relations in $\mathcal{B}$ and hence $\mathcal{B}, *, \mathcal{A}$ depend on the choice of the cilia at the vertices $x$. While this should not disturb us as far as the "unphysical" algebra $\mathcal{B}$ is concerned, we want the observable algebra $\mathcal{A}$ with $*$-operation $*$ to be defined on the graph $G$ rather than on a ciliated graph $G_{c i l}$.

Proposition 12 Suppose that $G_{c i l}$ and $G_{c i l^{\prime}}$ are two ciliated graphs which differ only by their ciliations. Then $\mathcal{A}\left(G_{c i l}\right) \cong \mathcal{A}\left(G_{c i l^{\prime}}\right)$ as $*$-algebras.

Proof: Let us consider an elementary move when the eyelash position changes at one vertex $x \in S$ for one step. This means that the smallest link incident to $x$ (in the ciliated graph $G_{c i l}$ ) becomes the largest link incident to $x$ in $G_{c i l^{\prime}}$. This link will be denoted by $i$. We agree to use $U_{\alpha}^{I}(j)$ for generators in $\mathcal{B}\left(G_{c i l}\right)=\mathcal{B}$ and $\hat{U}_{\alpha}^{I}(j)$ for generators of $\mathcal{B}\left(G_{c i l^{\prime}}\right)=\mathcal{B}^{\prime}$. If $F \in \mathcal{B}$ we write $\hat{F}$ to denote the corresponding element in $\mathcal{B}^{\prime}$ where all generators $U_{\alpha}^{I}(j)$ have been replaced by $\hat{U}_{\alpha}^{I}(j)$. The only effect of the different position of eyelashes is that the relations

$$
U_{\alpha}^{I}(i) U_{\beta}^{J}(j)=U_{\gamma}^{J}(j) U_{\delta}^{I}(i)\left(\tau_{\delta \alpha}^{I, i} \otimes \tau_{\gamma \beta}^{J, j}\right)\left(R_{x}\right)
$$

which hold for all links $j \neq i$ on $G_{c i l}$ incident to $x$ are substituted by

$$
\hat{U}_{\alpha}^{I}(i) \hat{U}_{\beta}^{J}(j)=\hat{U}_{\gamma}^{J}(j) \hat{U}_{\delta}^{I}(i)\left(\tau_{\delta \alpha}^{I, i} \otimes \tau_{\gamma \beta}^{J, j}\right)\left(R_{x}^{\prime-1}\right) .
$$

Observables in $\mathcal{B}$ are obtained as linear combinations of

$$
A=F_{\mu} U_{\alpha}^{I}(i) C_{\mu \alpha} .
$$

Here $F_{\mu}$ is a tuple of elements in $\mathcal{B}$ generated by $U_{\alpha}^{I}(j), \pm j \neq i$, and $F_{\mu}$ is supposed to transform covariantly according to the representation $\tau$ of $\mathcal{G} . C_{\mu \alpha}$ 
are complex numbers chosen in such a way that $A$ is invariant. Let $A^{\prime}$ be a second observable in $\mathcal{B}$ which is written in the same form with a tuple $F_{\nu}^{\prime}$ transforming according to the representation $\tau^{\prime}$, i.e. $A^{\prime}=F_{\nu}^{\prime} U_{\beta}^{J}(i) C_{\nu \beta}^{\prime}$. The product $A A^{\prime}$ defines coefficients $\tilde{C}_{\mu \nu \alpha \beta}$ such that

$$
A A^{\prime}=F_{\mu} F_{\nu}^{\prime} U_{\alpha}^{I}(i) U_{\beta}^{J}(i) \tilde{C}_{\mu \nu \alpha \beta}
$$

If we perform the same calculation in $\mathcal{B}^{\prime}$ we obtain

$$
\hat{A} \hat{A}^{\prime}=\hat{F}_{\mu} \hat{F}_{\rho}^{\prime} \hat{U}_{\gamma}^{I}(i) \hat{U}_{\beta}^{J}(i)\left(\tau_{\gamma \alpha}^{I, i} \otimes \tau_{\rho \nu}^{\prime}\right)\left(R_{x} R_{x}^{\prime}\right)^{-1} \tilde{C}_{\mu \nu \alpha \beta}
$$

This basically follows from proposition 6 and the influence of the different positions of the eyelash. Using the intertwining properties of the coefficients $\tilde{C}$, the equation $(\mathcal{S} \otimes i d) R=R^{-1}$, relations $(3.6)$ and functoriality on the link $i$ the product $\hat{A} \hat{A}^{\prime}$ can be rewritten as

$$
\begin{aligned}
\hat{A} \hat{A}^{\prime} & =\hat{F}_{\mu} \hat{F}_{\nu}^{\prime} \hat{U}_{\gamma}^{I}(i) \hat{U}_{\delta}^{J}(i)\left(\tau_{\gamma \alpha}^{I, i} \otimes \tau_{\delta \beta}^{J, j}\right)\left(R_{x}^{\prime} R_{x}\right) \tilde{C}_{\mu \nu \alpha \beta} \\
& =\sum_{K} \hat{F}_{\mu} \hat{F}_{\nu}^{\prime} \hat{U}_{\gamma}^{K}(i) C^{a}\left[\begin{array}{lll}
I & J & K \\
\alpha & \beta & \gamma
\end{array}\right]^{i} \tilde{C}_{\mu \nu \alpha \beta} \frac{v_{I} v_{J}}{v_{K}}
\end{aligned}
$$

In the last line $v_{I}=\tau_{x}^{I}\left(v_{x}\right)$ etc. . In other words, the map $E: \mathcal{A} \mapsto \mathcal{A}^{\prime}$ given by

$$
E\left(F_{\mu} U_{\alpha}^{I}(i) C_{\mu \alpha}\right)=\hat{F}_{\mu} \hat{U}_{\alpha}^{I}(i) C_{\mu \alpha} v_{I}^{-1}
$$

is an automorphism, i.e. $E\left(A A^{\prime}\right)=E(A) E\left(A^{\prime}\right)$. Let us finally check that this automorphism is compatible with the $*$-operation on observables.

$$
\begin{aligned}
E(A)^{*} & =\left(\hat{F}_{\mu} \hat{U}_{\alpha}^{I}(i) C_{\mu \alpha} v_{I}^{-1}\right)^{*} \\
& =\overline{C_{\mu \alpha}} v_{I} \theta\left(\hat{U}_{\alpha}^{I}(i)\right) \theta\left(\hat{F}_{\mu}\right) \\
& =\hat{F}_{\mu}^{\prime} \hat{U}_{\alpha}^{\bar{I}}(i) \tilde{C}_{\mu \alpha} v_{I}
\end{aligned}
$$

The last row is again meant to define the coefficients $\tilde{C}$ and the tuple $\hat{F}_{\mu}^{\prime}$. On the other hand we have

$$
\begin{aligned}
E\left(A^{*}\right) & =E\left(F_{\rho}^{\prime} U_{\beta}^{\bar{I}}(i)\left(\tau_{\beta \alpha}^{\bar{I}, i} \otimes \tau_{\rho \mu}^{\prime}\right)\left(R_{x} R_{x}^{\prime}\right) \tilde{C}_{\mu \alpha}\right) \\
& =E\left(F_{\nu}^{\prime} U_{\alpha}^{\bar{I}}(i) \tilde{C}_{\mu \alpha}\right) v_{I}^{2} \\
& =\hat{F}_{\nu}^{\prime} \hat{U}_{\alpha}^{\bar{I}}(i) \tilde{C}_{\mu \alpha} v_{I}
\end{aligned}
$$

So we conclude that $E\left(A^{*}\right)=E(A)^{*}$, i.e. $E$ defines a *automorphism between the algebras $\mathcal{A}$ and $\mathcal{A}^{\prime}$. Since two arbitrary ciliations of the graph $G$ can be obtained from each other by a series the elementary moves considered in this proof, we established the independence of the eyelash. Let us add that the isomorphisms between algebras constructed starting from the same graph with different ciliations are canonical. They are completely defined by the pair which consists of the graph and the symmetry Hopf algebra. 


\section{The regular representation}

Let us finally construct the regular representations of the lattice algebra $\mathcal{B}$ and the subalgebra $\mathcal{A}$ of observables. Both will act by multiplication operators on a space $\mathcal{F}$. Elements in $\mathcal{F}$ have an interpretation as "functions" on the (noncommutative) space of lattice connections. As an algebra, $\mathcal{F}$ is generated by a set of "coordinate functions" $u_{\alpha}^{I}(i)$. The elements of the gauge symmetry $\mathcal{G}$ act on $\mathcal{F}$ as generalized derivations. Using the $*$-operation on $\mathcal{B}$ and a generalized multidimensional Haar measure we will be able to define a scalar product on $\mathcal{F}$.

\subsection{The definition of $\mathcal{F}$}

In our present context it is obvious how to define the algebra $\mathcal{F}$ of "functions" on the space of lattice connections. So instead of giving a lengthy construction which works also in the more general cases considered below, we present an ad hoc definition for $\mathcal{F}$ and check that it carries the announced representation of $\mathcal{B}$.

Definition 13 (Algebra $\mathcal{F}$ ) The algebra $\mathcal{F}=\mathcal{F}\left(G_{\text {cil }}\right)$ is an associative unital algebra with unit $\Omega$. It is generated by elements $u_{\alpha}^{I}(i), i \in L$, subject to the relations

$$
\begin{aligned}
u_{\alpha}^{I}(i) \cdot u_{\beta}^{J}(i) & =\sum u_{\gamma}^{K}(i) C^{a}\left[\begin{array}{ccc}
I & J & K \\
\alpha & \beta
\end{array}\right]^{i}, \\
u_{\alpha}^{I}(-i) & =u_{\beta}^{\bar{I}}(i) \mu_{\beta \alpha}^{I, i}, \\
u_{\alpha}^{I}(i) \cdot u_{\beta}^{J}(j) & =u_{\delta}^{J}(j) \cdot u_{\gamma}^{I}(i)\left(\tau_{\gamma \alpha}^{I, i} \otimes \tau_{\delta \beta}^{J, j}\right)(R),
\end{aligned}
$$

for $i \leq j$ or if $i, j$ have no common endpoints.

We immediately recognize $\mathcal{F}$ to be our old algebra $\left\langle U_{\alpha}^{I}(i)\right\rangle$. However, this isomorphism is a mere coincidence. In the more general framework of quasi-Hopf algebras, the analogue of $\mathcal{F}$ turns out to be non-associative and consequently cannot be isomorphic to any subalgebra of the associative algebra $\mathcal{B}$. This remark might seem too prospective, but it should at least explain why we decided to give an independent definition of $\mathcal{F}$.

Because of their interpretation as functions in the non-commutative coordinates $u=\left(u_{\alpha}^{I}(i)\right)$, we will often use symbols $\psi(u), \phi(u)$ for elements in $\mathcal{F}$. A representation $\pi$ of $\mathcal{B}$ on $\mathcal{F}$ is defined by the following relations

$$
\begin{aligned}
\pi\left(U_{\alpha}^{I}(i)\right) \psi(u) & =u_{\alpha}^{I}(i) \cdot \psi(u), \\
\pi(\xi)\left(\psi_{1}(u) \cdot \psi_{2}(u)\right) & =\sum \pi\left(\xi_{\sigma}^{1}\right) \psi_{1}(u) \cdot \pi\left(\xi_{\sigma}^{2}\right) \psi_{2}(u), \\
\pi(\xi) u_{\alpha}^{I}(i)=u_{\beta}^{I}(i) \tau_{\beta \alpha}^{I, i}(\xi) & , \quad \pi(\xi) \Omega=\Omega \epsilon(\xi)
\end{aligned}
$$

for all $\psi(u), \psi_{1}(u), \psi_{2}(u) \in \mathcal{F}$. The first equation means that elements $U_{\alpha}^{I}(i)$ act as multiplication operators on $\mathcal{F}$. The last two lines specify the action of 
the gauge symmetry $\mathcal{G}$. Because of relation (6.5) one says that elements $\xi$ of the quantum symmetry act as generalized derivations on the algebra $\mathcal{F}$.

We see that the operators $\pi\left(U_{\alpha}^{I}(i)\right)$ generate $\mathcal{F}$ from the "constant function" $\Omega$. In particular $u_{\alpha}^{I}(i)=\pi\left(U_{\alpha}^{I}(i)\right) \Omega$. Given an element $\psi(u) \in \mathcal{F}$, its "generator" $\Psi(U) \in \mathcal{B}$ (i.e. $\psi(u)=\pi(\Psi(U)) \Omega$ ) is nearly unique. The only freedom in the choice of $\Psi(U)$ comes from the possibility to multiply from the right by factors $\xi \in \mathcal{G}, \epsilon(\xi)=1$, without changing the generated element $\psi(u) \in \mathcal{F}$.

A tuple $\psi_{\alpha}(u)$ of elements in $\mathcal{F}$ is said to transform covariantly according to the representation $\tau$ of $\mathcal{G}$ if

$$
\pi(\xi) \psi_{\alpha}(u)=\psi_{\beta}(u) \tau_{\beta \alpha}(\xi) .
$$

$\phi(u) \in \mathcal{F}$ is invariant, if $\pi(\xi) \phi(u)=\phi(u) \epsilon(\xi)$. Invariant elements $\phi(u) \in \mathcal{F}$ generate a subalgebra $\mathcal{F}^{\text {inv }}$ of $\mathcal{F}$. This subalgebra carries a representation of the algebra $\mathcal{A}$ of observables (the restriction of $\pi$ to the algebra of invariants $\mathcal{A}) . \mathcal{F}^{i n v}$ is the "algebra of functions" on the moduli space of connections.

\subsection{A scalar product on $\mathcal{F}$}

It is our aim to construct a scalar product on $\mathcal{F}$, i.e. for "functions" on the space of connections. The procedure mimics the classical situation. The main ingredient is a multidimensional Haar measure $\omega$, which allows to compute integrals of arbitrary functions on the space of connections. The scalar product of two functions $\psi_{i}(u)=\Psi_{i}(U) \Omega, i=1,2$ is then obtained as $\left\langle\psi_{2}(u) \mid \psi_{1}(u)\right\rangle \equiv$ $\omega\left(\Psi_{2}(U)^{*} \Psi_{1}(U)\right)$.

Instead of defining a functional directly on the algebra $\mathcal{F}$, we prefer to work with a linear map $\omega: \mathcal{B} \mapsto \mathbf{C}$. The relation to the multidimensional Haar measure will be apparent. By proposition 5, a linear functional on $\omega$ is specified when we prescribe the values it has on elements of the form (4.10). In the case of $\omega$ we want $\omega(e)=1$ and

$$
\omega\left(U_{\alpha_{1}}^{I_{1}}\left(i_{1}\right) \ldots U_{\alpha_{n}}^{I_{n}}\left(i_{n}\right) \xi\right)=\epsilon(\xi) \delta_{I_{1}, 0} \ldots \delta_{I_{n}, 0} .
$$

For this to be well defined it is essential that every link appears only once and only in one orientation among the links $i_{\nu}$. Some properties of $\omega$ are obvious. We state them here without proof.

$$
\begin{aligned}
\frac{\omega(\xi F)}{\omega(F)} & =\omega(F) \epsilon(\xi) \\
& \omega\left(F^{*}\right)
\end{aligned}
$$

for all $\xi \in \mathcal{G}, F \in \mathcal{B}$.

The interpretation of $\omega$ as a multidimensional Haar measure uses the correspondence between elements $\psi(u) \in \mathcal{F}$ and their generators $\Psi(U)$. Since $\omega$ depends on $\xi$ only through the value $\epsilon(\xi), \omega_{h}(\psi(u)) \equiv \omega(\Psi(U))$ does make sense. Relation (6.7) is the usual invariance $\omega_{h}(\pi(\xi) \psi(u))=\omega_{h}(\psi(u)) \epsilon(\xi)$ of the multidimensional Haar measure. 
Definition (6.6) has a fundamental drawback. Usually it requires an enormous calculation to bring an arbitrary element in $\mathcal{B}$ into the form (4.10). However there is a recursive way to calculate $\omega$. Once all elements assigned to a given link $i,-i$ are gathered, integration over these variables can be performed. The formal expression is

$$
\omega\left(F U_{\alpha}^{I}(i) G\right) \equiv \omega(F G) \omega\left(U_{\alpha}^{I}(i)\right)=\omega(F G) \delta_{I, 0}
$$

for all $F, G$ generated by $U_{\beta}^{J}(j), j \neq i,-i$ and elements $\xi \in \mathcal{G}$.

Let us practise the calculation of the functional $\omega$ in a simple but fundamental example.

Lemma 14 With the quantum dimension $d_{J}=\operatorname{Tr}\left(\tau^{J}\left(\mathcal{S}_{*}\left(u_{*}\right) v_{*}^{-1}\right)\right)$ and $v_{I}=$ $\tau^{I}\left(v_{*}\right)$ we have

$$
\omega\left(\theta\left(U_{\alpha}^{I}(i)\right) U_{\beta}^{I}(i)\right)=v_{I}^{-2} \omega\left(\eta_{\alpha \gamma}^{I,-i} U_{\gamma}^{I}(i) U_{\beta}^{I}(i)\right)=\delta_{\alpha, \beta} \frac{v_{I}^{-1}}{d_{J}} .
$$

PRoOF: The simplest proof for this formula makes use of the invariance (6.7) of $\omega$. The latter can be reformulated into the following intertwining property of the matrix $\Omega_{\alpha \beta}^{I, i}=\omega\left(\theta\left(U_{\alpha}^{I}(i)\right) U_{\beta}^{I}(i)\right)$.

$$
\tau_{\alpha \beta}^{I, i}(\xi) \Omega_{\beta \gamma}^{I, i}=\Omega_{\alpha \beta}^{I, i} \tau_{\beta \gamma}^{I, i}(\xi) \text { for all } \xi \in \mathcal{G} .
$$

Since $\tau^{I, i}$ is irreducible, we obtain that $\Omega_{\alpha \beta}^{I, i}=\lambda^{I, i} \delta_{\alpha, \beta}$. To calculate the complex number $\lambda^{I, i}$ we multiply this equation with $\left(\eta^{I,-i}\right)_{\beta \alpha}^{-1}$ and sum over $\alpha, \beta$. The result is

$$
\begin{aligned}
\lambda^{I, i} \operatorname{Tr}\left(\left(\eta^{I,-i}\right)^{-1}\right) & =\sum\left(\eta^{I,-i}\right)_{\beta \alpha}^{-1} \omega\left(\theta\left(U_{\alpha}^{I}(i)\right) U_{\beta}^{I}(i)\right) \\
& =\sum v_{I}^{-2} \omega\left(U_{\beta}^{I}(-i) U_{\beta}^{I}(i)\right)=v_{I}^{-2} \delta_{I} .
\end{aligned}
$$

In this calculation we used the formula (4.15) for $\theta$, the invariance (6.7) of $\omega$ and the functoriality on the link $i(4.3)$. $\delta_{I}$ is the ordinary dimension of the representation $\tau_{*}^{I}$, i.e. $\delta_{I}=\operatorname{dim}\left(V^{I}\right)$. Since $\operatorname{Tr}\left(\left(\eta^{I,-i}\right)^{-1}\right)=\delta_{I} \operatorname{Tr}\left(\tau^{I}\left(\mathcal{S}_{*}\left(u_{*}^{-1}\right)\right)\right)=$ $v_{I}^{-1} \delta_{I} d_{I}$ we infer that $\lambda^{I, i}=v_{I}^{-1} / d_{I}$.

After this warm up we can address more complicated examples. Recall that we plan to evaluate $\omega\left(\left(\Psi_{2}(U)\right)^{*} \Psi_{1}(U)\right)$ for elements $\Psi_{\nu}(U) \in \mathcal{B}$. This motivates to calculate

$$
\omega\left(\left(U_{\alpha_{1}}^{I_{1}}\left(i_{1}\right) \ldots U_{\alpha_{n}}^{I_{n}}\left(i_{n}\right)\right)^{*} U_{\beta_{1}}^{I_{1}}\left(i_{1}\right) \ldots U_{\beta_{n}}^{I_{n}}\left(i_{n}\right)\right)
$$

for a set of links $i_{\nu}$ which satisfies the assumption of proposition 5. We write the $*$-operation as $\sigma_{\kappa} \circ \theta$. The conjugation with $\kappa$ effects the value of expression (6.10) in a simple way. The $\kappa^{-1}$ to the left gives a factor $\epsilon(\kappa)=1$ because of eq. $(\overline{6.7})$. So there remains only one $\kappa$ in the middle. It can be removed from the argument of $\omega$ by means of the covariance relation (4.1) and invariance of the 
generalized Haar measure $\omega$. After these manipulations $\sigma_{\kappa}$ is seen to contribute with a factor $\kappa$ evaluated in the tensor product of representations $\tau^{I_{1}, i_{1}}$ through $\tau^{I_{n}, i_{n}}$. Now we are left with $\theta$. Since $\theta$ is an anti-automorphism, we find that all variables attached to the link $i_{1},-i_{1}$ are already gathered in the middle of the functional. So the "integration" can be performed and results in an expression where variables on $i_{2},-i_{2}$ appear together. This continues until everything is reduced to the unit element $e$. Evaluation on a single link is an application of the lemma. All one has to care about are the factors involving the $R$-matrix which come with the anti-automorphism $\theta$. The value of (6.10) is

$$
\left(\tau_{\alpha_{1} \beta_{1}}^{I_{1}, i_{1}} \otimes \ldots \otimes \tau_{\alpha_{n} \beta_{n}}^{I_{n}, i_{n}}\right)\left(R^{(n-1)^{\prime}} \Delta^{(n-1)}(\kappa)\right) \prod_{\nu} v_{I_{\nu}}^{-1} d_{I_{\nu}}^{-1}
$$

where the ' means that one uses $\Delta^{\prime}$ instead of $\Delta$ in definition 5.2

Theorem 15 (scalar product) Let $\Psi_{\nu}(U), \nu=1,2$ be elements in $\mathcal{B}$ which generate the states $\psi_{\nu}(u)$ in the sense that $\psi_{\nu}(u)=\pi\left(\Psi_{\nu}(U)\right)|0\rangle$. The bilinear form

$$
<\psi_{1}(u) \mid \psi_{2}(u)>\equiv \omega\left(\Psi_{1}(U)^{*} \Psi_{2}(U)\right)
$$

defines a scalar product on $\mathcal{F}$ if and only if the quantum dimensions $d_{I}$ satisfy $d_{I}>0$ for all labels $I$ (and provided that the condition 3.1 ) is satisfied). In particular, the assumption on $d_{I}$ guarantees that $\omega$ is a positive linear functional on $\mathcal{A}$.

Remark: $\omega$ does not define a positive functional on $\mathcal{B}$ or $\left\langle U_{\alpha}^{I}(i)>\subset \mathcal{B}\right.$. For $\mathcal{B}$ this is due to the fact that $\mathcal{G} \subset \mathcal{B}$. The properties of $\omega$ show $\omega\left(\xi^{*} \xi\right)=\epsilon\left(\xi^{*}\right) \epsilon(\xi)=$ 0 , whenever $\epsilon(\xi)=0$. If $\mathcal{G}$ is not trivial, it has nonzero elements $\xi$ with $\epsilon(\xi)=0$ and this obviously violates positivity. On the other hand, $F^{*} \notin<U_{\alpha}^{I}(i)>$ can happen, even if $F \in<U_{\alpha}^{I}(i)>$. Consequently, the ${ }^{*}$-operation on $\mathcal{B}$ does not restrict to $\left\langle U_{\alpha}^{I}(i)>\right.$ so that there is no way to formulate positivity of $\omega$ on $<U_{\alpha}^{I}(i)>$.

PROOF OF THE THEOREM: We remarked before that $\langle\mid\rangle$ is well defined. It is linear in the second argument and anti-linear in the first. The property

$$
\overline{<\psi_{1}(u) \mid \psi_{2}(u)>}=<\psi_{2}(u) \mid \psi_{1}(u)>
$$

holds due to relation (6.8). Positivity is a consequence of the formula (6.11) and our assumption 3.13 . If $C_{\beta_{1} \ldots \beta_{n}}^{\beta}$ has the intertwining property

$$
\left(\tau_{\alpha_{1} \beta_{1}}^{I_{1}, i_{1}} \otimes \cdots \otimes \tau_{\alpha_{n} \beta_{n}}^{I_{n}, i_{n}}\right)(\xi) C_{\beta_{1} \ldots \beta_{n}}^{\beta}=\tau_{\beta \alpha}(\xi) C_{\alpha_{1} \ldots \alpha_{n}}^{\alpha},
$$

with $\tau$ being some irreducible representation of $\mathcal{G}$, relation (3.13) implies that (no summation over $\alpha$ )

$$
\overline{C_{\alpha_{1} \ldots \alpha_{n}}^{\alpha}}\left(\tau_{\alpha_{1} \beta_{1}}^{I_{1}, i_{1}} \otimes \ldots \otimes \tau_{\alpha_{n} \beta_{n}}^{I_{n}, i_{n}}\right)\left(R^{(n-1)^{\prime}}\right) C_{\beta_{1} \ldots \beta_{n}}^{\alpha}=\overline{C_{\alpha_{1} \ldots \alpha_{n}}^{\alpha}} C_{\alpha_{1} \ldots \alpha_{n}}^{\alpha} \kappa_{\tau}^{-1} \prod_{\nu} v_{I_{\nu}}
$$


Here we used $\kappa_{\tau} \equiv \tau(\kappa)$ and $\kappa_{J}^{2}=v_{J}$. We see that the complex phases $v_{I_{\nu}}$ in the last expression cancel the phases in (6.11), while the factor $\kappa_{\tau}^{-1}$ cancels the contribution from $\Delta^{(n-1)}(\kappa)$. With $F=U_{\alpha_{1}}^{I_{1}}\left(i_{1}\right) \ldots U_{\alpha_{n}}^{I_{n}}\left(i_{n}\right) C_{\alpha_{1} \ldots \alpha_{n}}^{\alpha}$ we obtain

$$
\omega\left(F^{*} F\right)=\overline{C_{\alpha_{1} \ldots \alpha_{n}}^{\alpha}} C_{\alpha_{1} \ldots \alpha_{n}}^{\alpha} \prod_{\nu} \frac{1}{d_{I_{\nu}}}
$$

This is positive, if the quantum dimensions are.

There is one important remark we have to make at this point. Everything we did so far works for $U_{q}(\mathcal{G})$ at generic values of the deformation parameter $q$, since $U_{q}(\mathcal{G})$ is semisimple in these cases. So it may seem that we just quantized Chern-Simons for arbitrary (non-integer) values of the level $k$. However, the positivity of the scalar product is conditional on the positivity of the quantum dimensions $d_{J}$. The latter fails to hold for many representations of $U_{q}(\mathcal{G})$, so that we recover the usual quantization condition. In the next section we will deal with the roots of unity. It is shown that Chern Simons can be quantized only for primitive roots of unity, i.e. for integer values of $k$.

\section{Generalization to quasi Hopf algebras}

In this section we want to generalize our theory to cases in which the local symmetry $\mathcal{G}_{x}$ is a quasi-Hopf algebra. There are at least two motivations to do this. When we discussed the twist equivalence of the symmetry algebras $\mathcal{G}_{x}$ we saw that the co-associativity of $\Delta_{x}$ led to a severe constraint on the possible twist. One is tempted to remove this constraint and admit all possible unitary twist elements $F_{x}$ without caring about co-associativity. This is precisely what quasi-Hopf algebra are designed for. We will see that our observable algebra depends only on the "twist class" of the symmetry $\mathcal{G}_{x}$. To understand the second motivation we recall that the assumption about semisimplicity does not apply to the most interesting cases, e.g. $U_{q}\left(s l_{2}\right), q^{p}=1$. However, working with the semisimple "truncated" symmetry algebra $U_{q}^{T}\left(s l_{2}\right)$ introduced in [2], we can bypass this problem. By construction, the representation theory of $U_{q}^{T}\left(s l_{2}\right)$ coincides with the "physical" part of the representation theory of $U_{q}\left(s l_{2}\right)$. There is a price we have to pay for this: $U_{q}^{T}\left(s l_{2}\right)$ is no longer a Hopf algebra but only a (weak) quasi-Hopf algebra.

\subsection{Short reminder on quasi-Hopf algebras}

At this point we want to recall some of the defining features of (weak) quasiHopf algebras. Quasi-Hopf algebras have been introduced in [11]. The axioms stated there can be weakened to allow for "truncation" in the tensor product of representations. The resulting structures were called "weak quasi Hopf algebras" [2]. 
In comparison to Drinfel'd, we want to admit that $\Delta(e) \neq e \otimes e$, where $e$ is the unit element in an algebra $\mathcal{G}$. It still follows from the homomorphism property of $\Delta, \Delta(\xi \eta)=\Delta(\xi) \Delta(\eta)$, that $\Delta(e)$ is a projector $P$ in $\mathcal{G} \otimes \mathcal{G}$ and that this projector commutes with $\Delta(\xi)$ for all $\xi \in \mathcal{G}$. Consequently, the linear map $\left(\tau \otimes \tau^{\prime}\right)(P)$ projects onto a subrepresentation of $\tau \otimes \tau^{\prime}$. If $\left(\tau \otimes \tau^{\prime}\right)(P) \neq i d$, the tensor product $\tau \otimes \tau^{\prime}$ is said to be truncated.

The generalization of Drinfel'ds axioms to the "weak" case is almost straightforward. As in 11 we demand that an element $\varphi \in \mathcal{G} \otimes \mathcal{G} \otimes \mathcal{G}$ is given which implements (weak) quasi-co-associativity of the coproduct,

$$
\varphi(\Delta \otimes i d) \Delta(\xi)=(i d \otimes \Delta) \Delta(\xi) \varphi \text { for all } \xi \in \mathcal{G} .
$$

Because of the truncation, this element $\varphi$ cannot be invertible in general. So invertibility is substituted by a weaker assumption on the existence of a quasiinverse, still denoted by $\varphi^{-1}$, such that

$$
\begin{aligned}
\varphi \varphi^{-1}=(i d \otimes \Delta) \Delta(e) & , \quad \varphi^{-1} \varphi=(\Delta \otimes i d) \Delta(e), \\
(i d \otimes \epsilon \otimes i d)(\varphi) & =\Delta(e) .
\end{aligned}
$$

The statement that $\varphi^{-1}$ is a quasi-inverse of $\varphi$ means that $\varphi \varphi^{-1} \varphi=\varphi$. Evaluated with the representations $\tau, \tau^{\prime}, \tau^{\prime \prime}$, the re-associator $\varphi$ furnishes an intertwiner between the representations $\left(\tau \otimes \tau^{\prime}\right) \otimes \tau^{\prime \prime}$ and $\tau \otimes\left(\tau^{\prime} \otimes \tau^{\prime \prime}\right)$. This means that the tensor product of representations is associative up to equivalence.

Similarly we do not demand that the element $R$ be invertible. Instead it should have a quasi-inverse $R^{-1}$ such that

$$
R R^{-1}=\Delta^{\prime}(e) \quad, \quad R^{-1} R=\Delta(e) .
$$

This is sufficient to implement the equivalence between representations $\tau \otimes \tau^{\prime}$ and $\tau^{\prime} \otimes \tau$.

Following Drinfeld we postulate several relations between $\Delta, R$, and $\varphi$.

$$
\begin{aligned}
(i d \otimes i d \otimes \Delta)(\varphi)(\Delta \otimes i d \otimes i d)(\varphi) & =(e \otimes \varphi)(i d \otimes \Delta \otimes i d)(\varphi)(\varphi \otimes e), \\
(i d \otimes \Delta)(R) & =\varphi_{231}^{-1} R_{13} \varphi_{213} R_{12} \varphi^{-1} \\
(\Delta \otimes i d)(R) & =\varphi_{312} R_{13} \varphi_{132}^{-1} R_{23} \varphi .
\end{aligned}
$$

We used the standard notation. If $\mathrm{s}$ is any permutation of 123 and $\varphi=\sum \varphi_{\sigma}^{1} \otimes$ $\varphi_{\sigma}^{2} \otimes \varphi_{\sigma}^{3}$ then

$$
\varphi_{s(1) s(2) s(3)}=\sum_{\sigma} \varphi_{\sigma}^{s^{-1}(1)} \otimes \varphi_{\sigma}^{s^{-1}(2)} \otimes \varphi_{\sigma}^{s^{-1}(3)} .
$$

Eqs. (7.4) imply validity of quasi Yang Baxter equations,

$$
R_{12} \varphi_{312} R_{13} \varphi_{132}^{-1} R_{23} \varphi=\varphi_{321} R_{23} \varphi_{231}^{-1} R_{13} \varphi_{213} R_{12},
$$


and this guarantees that $R$ together with $\varphi$ determines a representation of the braid group [1].

It is assumed that $(i d \otimes \epsilon \otimes i d)(\varphi)=\Delta(e)$. Similar relations for the action of the co-unit $\epsilon$ on other components of $\varphi$ and the components of $R$ follow from this.

All relations for the antipode $\mathcal{S}$ can be copied from Drinfel'd. It is supposed that there is an anti-automorphism $\mathcal{S}$ and two elements $\alpha, \beta \in \mathcal{G}$, such that

$$
\sum \mathcal{S}\left(\xi_{\sigma}^{1}\right) \alpha \xi_{\sigma}^{2}=\alpha \epsilon(\xi) \quad, \quad \sum \xi_{\sigma}^{1} \beta \mathcal{S}\left(\xi_{\sigma}^{2}\right)=\beta \epsilon(\xi)
$$

Moreover, the following relations are required to hold.

$$
\sum \varphi_{\sigma}^{1} \beta \mathcal{S}\left(\varphi_{\sigma}^{2}\right) \alpha \varphi_{\sigma}^{3}=e, \quad \sum \mathcal{S}\left(\phi_{\sigma}^{1}\right) \alpha \phi_{\sigma}^{2} \beta \mathcal{S}\left(\phi_{s}^{3}\right)=e
$$

Here $\phi=\varphi^{-1}=\sum \phi_{\sigma}^{1} \otimes \phi_{\sigma}^{2} \otimes \phi_{\sigma}^{3}$.

Everything we said about ${ }^{*}$-structures in section 3 remains true. But we have to add two more requirements which describe the behaviour of the elements $\varphi$ and $\alpha, \beta$ with respect to conjugation. One can check that the equations

$$
\varphi^{*}=\sum \varphi_{\sigma}^{3 *} \otimes \varphi_{\sigma}^{2 *} \otimes \varphi_{\sigma}^{1 *}=\varphi, \alpha^{*}=\beta
$$

are consistent with the other relations which involve $\varphi$ or $\alpha, \beta$.

For Hopf-algebras it is well known that $\Delta(\phi)=(\mathcal{S} \otimes \mathcal{S}) \Delta^{\prime}\left(\mathcal{S}^{-1}(\phi)\right)$. A generalization of this fact was already noticed by Drinfel'd [11. To state his observation we introduce the following notations.

$$
\begin{aligned}
\gamma= & \sum \mathcal{S}\left(U_{\sigma}\right) \alpha V_{\sigma} \otimes \mathcal{S}\left(T_{\sigma}\right) \alpha W_{\sigma} \\
\text { with } & \sum T_{\sigma} \otimes U_{\sigma} \otimes V_{\sigma} \otimes W_{\sigma}=(\varphi \otimes e)(\Delta \otimes i d \otimes i d)\left(\varphi^{-1}\right), \\
f= & \sum(\mathcal{S} \otimes \mathcal{S})\left(\Delta^{\prime}\left(\phi_{p}^{1}\right)\right) \gamma \Delta\left(\phi_{p}^{2} \beta \mathcal{S}\left(\phi_{p}^{3}\right)\right), \\
\text { with } \quad & \phi=\varphi^{-1}=\sum \xi_{p}^{1} \otimes \xi_{p}^{2} \otimes \xi_{p}^{3} .
\end{aligned}
$$

Drinfel'd proved in [1] that the element $f$ satisfies

$$
\begin{aligned}
f \Delta(\xi) f^{-1} & =(\mathcal{S} \otimes \mathcal{S}) \Delta^{\prime}\left(\mathcal{S}^{-1}(\xi)\right) \text { for all } \xi \in \mathcal{G} \\
\gamma & =f \Delta(\alpha)
\end{aligned}
$$

The first equation asserts that $f$ "intertwines" between the co-product $\Delta$ and the combination of $\Delta$ and $\mathcal{S}$ on the right hand side.

Nontriviality of $\varphi, \alpha$ effects the expression for the element $u \in \mathcal{G}$. The relations (3.3) now hold for (cp. 22])

$$
u=\sum \mathcal{S}\left(\phi_{\sigma}^{2} \beta \mathcal{S}\left(\phi_{\sigma}^{3}\right)\right) \mathcal{S}\left(r_{\tau}^{2}\right) \alpha r_{\tau}^{1} \phi_{\sigma}^{1}
$$


with $\phi=\varphi^{-1}=\sum \phi_{\sigma}^{1} \otimes \phi_{\sigma}^{2} \otimes \phi_{\sigma}^{3}$. Altschuler and Coste also introduced the concept of ribbon quasi-Hopf algebras. As before, $u \mathcal{S}(u)$ is central and a "square root" $v$ with properties (3.5.3.6) it called ribbon element.

Examples of weak ribbon quasi-Hopf algebras $\mathcal{G}$ are canonically associated with $U_{q}\left(s l_{2}\right)$ with $q$ a root of unity. As an algebra $\mathcal{G} \equiv U_{q}^{T}\left(s l_{2}\right) \equiv U_{q}\left(s l_{2}\right) / \mathcal{J}$, where $\mathcal{J}$ is the ideal which is annihilated by all the physical representations $\tau^{I}, 2 I=0 \ldots p-2$, of $U_{q}\left(s l_{2}\right) . \mathcal{U}_{q}^{T}\left(s l_{2}\right)$ is semisimple, its representations are fully reducible, and the irreducible ones are precisely the physical representations of $U_{q}\left(s l_{2}\right)$. Let

$$
u(I, J)=\min \{|I+J|, p-2-I-J\}
$$

and let $P_{I J}$ be the projector on the physical subrepresentations $\mathrm{K},|I-J| \leq K \leq$ $u(I, J)$ of the tensor product $\tau^{I} \otimes_{q} \tau^{J}$ of $U_{q}\left(s l_{2}\right)$ representations. There exists $P \in \mathcal{G}$ such that $P_{I J}=\left(\tau^{I} \otimes \tau^{J}\right)(P)$. The coproduct in $U_{q}^{T}\left(s l_{2}\right)$ is determined in terms of the coproduct $\Delta_{q}$ in $U_{q}\left(s l_{2}\right)$ as

$$
\Delta(\xi)=P \Delta_{q}(\xi),
$$

hence $\Delta(e)=P \neq e \otimes e$. This coproduct specifies a tensor product $\otimes$ which is equal to the truncated tensor product of physical $U_{q}\left(s l_{2}\right)$ representations. Thus

$$
\tau^{I} \otimes \tau^{J}=\bigoplus_{|I-J| \leq K \leq u(I J)} \tau^{K} .
$$

There exists an element $\varphi \in U_{q}^{T}\left(s l_{2}\right)^{\otimes_{3}}$ such that $\varphi^{I J K}=\left(\tau^{I} \otimes \tau^{J} \otimes \tau^{K}\right)(\varphi)$ imple truncated tensor products $\tau^{I} \otimes\left(\tau^{J} \otimes \tau^{K}\right)$ and $\left(\tau^{I} \otimes \tau^{J}\right) \otimes \tau^{K}$. The map $\varphi^{I J K}$ can be specified by its action on Clebsch Gordon intertwiners, together with the condition $\varphi=(i d \otimes \Delta) \Delta(e) \varphi$, viz.

$$
C(I P \mid L) C(J K \mid P)_{23} \varphi^{I J K}=\sum_{Q}\left\{\begin{array}{lll}
K & J & P \\
Q
\end{array}\right\}_{q} C(I J \mid Q) C(Q K \mid L)_{12},
$$

where $C\left(. . \mid\right.$.) denote the Clebsch Gordon maps and \{\} the 6J-symbols of $U_{q}\left(s l_{2}\right)$, $q^{p}=1$, evaluated for physical labels $I, J, K$. Summation over $Q$ is restricted to the physical representations.

The $R$-element of $U_{q}^{T}\left(s l_{2}\right) \otimes U_{q}^{T}\left(s l_{2}\right)$ is given in terms of the $R$-element $R_{q}$ for $U_{q}\left(s l_{2}\right)$ by

$$
R=R_{q} \Delta(e)=\Delta^{\prime}(e) R_{q},
$$

while antipode, ${ }^{*}$ and co-unit are the same as in $U_{q}\left(s l_{2}\right)$. One can show (cp. ref. [2]) that the defining properties of a weak quasitriangular quasi Hopf-*algebra are satisfied. The ${ }^{*}$-operation is of the type discussed above, i.e. $\Delta$ is a $*$-homomorphism provided that $(\xi \otimes \eta)^{*}=\eta^{*} \otimes \xi^{*}$. The ribbon element $v$ in $U_{q}\left(s l_{2}\right)$ survives the truncation and gives a ribbon element in $U_{q}^{T}\left(s l_{2}\right)$.

The truncation procedure described here can be generalized to other quantized enveloping algebras. We emphasize that the assumption (3.13) holds for all truncated quantized universal enveloping algebras associated with simple Lie algebras. 


\subsection{Results on quasi-quantum group gauge fields}

Our exposition will be restricted to the main results and those parts which deviate from the above theory for Hopf algebras.

A formulation of twist-equivalence involves the additional relations

$$
\begin{aligned}
\left(\iota_{x} \otimes \iota_{x} \otimes \iota_{x}\right)\left(\varphi_{x}\right) & =\left(e \otimes F_{x}^{-1}\right)\left(e \otimes \Delta_{*}\right)\left(F_{x}^{-1}\right) \varphi_{*}\left(\Delta_{*} \otimes e\right)\left(F_{x}\right)\left(F_{x} \otimes e\right) \\
\iota_{x}\left(\alpha_{x}\right) & =\sum \mathcal{S}_{*}\left(f_{x \sigma}^{1}\right) \alpha_{*} f_{x \sigma}^{2}
\end{aligned}
$$

with $f_{x \sigma}^{i}$ defined through $F_{x}=\sum f_{x \sigma}^{1} \otimes f_{x \sigma}^{2}$. Observe that the element $u_{x}$ introduced in $(\overline{7.12})$ is independent of the twist, i.e. $\iota_{x}\left(u_{x}\right)=u_{*}$. The same holds for the ribbon element $v_{x}$.

The discussion of gauge symmetry remains unchanged except from some minor points in defining the intertwiners at the end of section 3. First of all, while the definition of Clebsch Gordon maps $C_{x}^{a}[I J \mid K]$ at the sites $x \in S$ remain as before, the element $f$ constructed in eq. $(7.10)$ appears in the definition of the intertwiners

$$
C^{a}[I J \mid K]^{i} \equiv C_{y}^{a}[I J \mid K] \otimes{ }^{t}\left(\left(\tau_{x}^{I} \otimes \tau_{x}^{J}\right)\left(f_{x}^{\prime}\right) C_{x}^{a}[I J \mid K]^{*}\right)
$$

which are attached to the links $i \in L$. Of course we use the element (7.12) now to obtain the intertwiner $\eta^{I, i}$. The change in $\mu^{I, i}$ is slightly more subtle. In the quasi-Hopf case the right substitute is

$$
\mu^{I, i}=n^{I t_{2}} C[I \bar{I} \mid 0]^{i} \tau^{I, i}\left(\mathcal{S}^{-1}(\alpha)\right)\left(\eta^{I, i}\right)^{-1} .
$$

The last change we have to mention concerns the definition of quantum dimensions $d_{I}$. Their definition gets modified according to (cp. [22])

$$
d_{J} \equiv \operatorname{Tr}\left(\tau^{J}\left(\mathcal{S}_{*}\left(\beta_{*} u_{*}\right) v_{*}^{-1} \alpha_{*}\right) .\right.
$$

Elements $\kappa_{x} \in \mathcal{G}_{x}$ and the corresponding element $\kappa \in \mathcal{G}$ are defined as before.

The construction of the lattice algebra $\mathcal{B}$ is seriously effected by the generalization. To understand the major changes which occur when passing to quasiHopf algebras, it is crucial to notice that due to the lack of co-associativity, products of covariant elements are not covariant in general. This motivates the definition of "covariant products" [2].

Suppose that an algebra $\mathcal{B}$ contains a quasi- Hopf algebra $\mathcal{G}$ as subalgebra and that $\left(F_{\alpha}\right)_{\alpha \in I},\left(F_{\beta}^{\prime}\right)_{\beta \in I^{\prime}}$ transform covariantly according to representations $\tau$ and $\tau^{\prime}$ of $\mathcal{G}$ with dimensions $n$ and $n^{\prime}$ (in the sense of definition 4.5). Define the $\times$-product of the components by

$$
\left(F_{\alpha} \times F_{\beta}^{\prime}\right)=\sum_{\gamma \in I} \sum_{\delta \in I^{\prime}} F_{\gamma} F_{\delta}^{\prime}\left(\tau_{\gamma \alpha} \otimes \tau_{\delta \beta}^{\prime} \otimes i d\right)(\varphi) \in \mathcal{A} .
$$


Using the expansion $\varphi=\sum \varphi_{\sigma}^{1} \otimes \varphi_{\sigma}^{2} \otimes \varphi_{\sigma}^{3}$ the defining eq.(7.18) takes the form

$$
\left(F_{\alpha} \times F_{\beta}^{\prime}\right)=\sum_{\sigma} F_{\gamma} F_{\delta}^{\prime} \tau_{\gamma \alpha}\left(\varphi_{\sigma}^{1}\right) \tau_{\delta \beta}^{\prime}\left(\varphi_{\sigma}^{2}\right) \varphi_{\sigma}^{3}
$$

This exhibits the fact that the $\left(F_{\alpha} \times F_{b}^{\prime}\right)$ are complex linear combinations of terms $F_{\gamma} F_{\delta}^{\prime} \varphi_{\sigma}^{3}$ with coefficients $\varphi_{\sigma}^{3} \in \mathcal{G}$.

Proposition 16 (Properties of the $\times$-product) Let $\left(F_{\alpha}\right),\left(F_{\beta}^{\prime}\right)$ be specified as above and suppose that the unit $e \in \mathcal{G}$ is a unit element in $\mathcal{B}$. Then the $\times-$ product (7.18) has the following properties.

1. Eq.(7.18) can be inverted to recover ordinary products from covariant ones, viz.

$$
F_{\alpha} F_{\beta}^{\prime}=\sum\left(F_{\gamma} \times F_{\delta}^{\prime}\right)\left(\tau_{\gamma \alpha} \otimes \tau_{\delta \beta}^{\prime} \otimes i d\right)\left(\varphi^{-1}\right) .
$$

2. The tuple $F \times F^{\prime}=\left(F_{\alpha} \times F_{\beta}^{\prime}\right)$ transforms covariantly according to the tensor product representation $\tau \otimes \tau^{\prime}$ of $\mathcal{G}$. Hence we will often use the term covariant product instead of $\times$-product.

3. The $\times$-product is not associative. But it is quasi-associative in the following sense. If $F^{\prime \prime}=\left(F_{\gamma}^{\prime \prime}\right)$ transforms covariantly according to representations and $\tau^{\prime \prime}$ of $\mathcal{G}$ and $F, F^{\prime}$ as above, then

(i) $\left(\left(F_{\alpha} \times F_{\beta}^{\prime}\right) \times F_{\gamma}^{\prime \prime}\right)=\left(F_{\delta} \times\left(F_{\epsilon}^{\prime} \times F_{\kappa}^{\prime \prime}\right)\right)\left(\tau_{\delta \alpha} \otimes \tau_{\epsilon \beta}^{\prime} \otimes \tau_{\kappa \gamma}^{\prime \prime}\right)(\varphi)$

(ii) $\left(F_{\alpha} \times\left(F_{\beta}^{\prime} \times F_{\gamma}^{\prime \prime}\right)\right)=\left(\left(F_{\delta} \times F_{\epsilon}^{\prime}\right) \times F_{\kappa}^{\prime \prime}\right)\left(\tau_{\delta \alpha} \otimes \tau_{\epsilon \beta}^{\prime} \otimes \tau_{\kappa \gamma}^{\prime \prime}\right)\left(\varphi^{-1}\right)$.

4. If $G \in \mathcal{B}$ is $\mathcal{G}$-invariant then

$$
G \times F_{\alpha}=G F_{\alpha}, F_{a} \times G=F_{\alpha} G .
$$

All items in this proposition follow from the properties of the re-associator $\varphi$. Proofs are spelled out in [1].

Armed with the notion of covariant products the definition of the lattice algebra $\mathcal{B}$ is straightforward. We basically follow the rule to substitute all ordinary products between generators $U_{\alpha}^{I}(i)$ by $\times$ - products. So only functoriality and braid relations are concerned. To implement functoriality on the link we divide by the new relations

$$
\begin{aligned}
U_{\alpha}^{I}(i) \times U_{\beta}^{J}(i) & =\sum U_{\gamma}^{K}(i) C^{a}\left[\begin{array}{ccc}
I & J & K \\
\alpha & \beta & \gamma
\end{array}\right]^{i}, \\
U_{\alpha}^{I}(-i) & =U_{\beta}^{\bar{I}}(i) \mu_{\beta \alpha}^{I, i} .
\end{aligned}
$$

while the braid relations become

$$
U_{\alpha}^{I}(i) \times U_{\beta}^{J}(j)=U_{\gamma}^{J}(j) \times U_{\delta}^{I}(i)\left(\tau_{\delta \alpha}^{I, i} \otimes \tau_{\gamma \beta}^{J, j}\right)(R) .
$$


for $i \leq j$ or if $\mathrm{i}, \mathrm{j}$ have no common endpoints. These relations can be written in an alternative form involving again the ordinary (associative) product in $\mathcal{B}$. For example the braid relations can be formulated as

$$
U_{\alpha}(i) U_{\beta}(j)=U_{\gamma}(j) U_{\delta}(i)\left(\tau_{\delta \alpha}^{i} \otimes \tau_{\gamma \beta}^{j} \otimes i d\right)(\mathcal{R}),
$$

where $\mathcal{R}=\varphi_{213} R_{12} \varphi^{-1}$ is the element which is used to build up the representation of the braid group in the quasi-Hopf case [1].

The proposition 5 carries over to the more general case but is is often more convenient to use another set of linear generators built with the help of the covariant product. By proposition 16, elements

$$
U_{\alpha_{n}}^{I_{n}}\left(i_{n}\right) \times\left(\ldots \times\left(U_{\alpha_{2}}^{I_{2}}\left(i_{2}\right) \times U_{a_{1}}^{I_{1}}\left(i_{1}\right)\right) \ldots\right) \xi \quad \text { with } \quad n \geq 0, \xi \in \mathcal{G} .
$$

span $\mathcal{B}$. The brackets in this expression are necessary since the $\times$-product is not associative if $\varphi$ is non-trivial.

Observe that the possibility to move elements $\xi \in \mathcal{G}$ was an important ingredient in the Hopf-algebra case. However, the proof of proposition 4 relied on the co-associativity. Analogue formulas in context of quasi-Hopf algebras are more complicated.

Proposition 17 (right covariance) Let the element $w \in \mathcal{G} \otimes \mathcal{G}$ be defined by $w \equiv \sum \varphi_{\sigma}^{2} \mathcal{S}^{-1}\left(\varphi_{\sigma}^{1} \beta\right) \otimes \varphi_{\sigma}^{3}$ and $m \in \mathcal{G} \otimes \mathcal{G}$ similarly as $m \equiv \sum \mathcal{S}\left(\phi_{\sigma}^{1}\right) \alpha \phi_{\sigma}^{2} \otimes \phi_{\sigma}^{3}$ with the components $\phi_{\sigma}^{i}$ of $\phi=\varphi^{-1}$. Suppose that the tuple $\left(F_{\alpha}\right), F_{\alpha} \in \mathcal{B}$ transforms (right-) covariantly according to the representation $\tau$ of $\mathcal{G}$. Then the linear combination

$$
{ }_{\alpha} F \equiv F_{\beta}\left(\tau_{\beta \alpha} \otimes i d\right)(w)
$$

has the following properties.

1. ${ }_{\alpha} F$ transforms left-covariantly according to the representation $\tilde{\tau}$,

$$
{ }_{\alpha} F \xi=\left(\tilde{\tau}_{\alpha \beta} \otimes i d\right)(\Delta(\xi)){ }_{\beta} F
$$

for all $\xi \in \mathcal{G}$.

2. The transformation from right- to left-covariant elements can be inverted,

$$
F_{\alpha}=\left(\tilde{\tau}_{\alpha \beta} \otimes i d\right)(m)_{\beta} F .
$$

3. The passage (7.20) from right- to left covariant elements is consistent with braid relations. Suppose that $F_{\beta}^{\prime}$ is a second right-covariant multiplet transforming covariantly according to the representation $\tau^{\prime}$ and that $F_{\alpha}, F_{\beta}^{\prime}$ satisfy braid relations

$$
F_{\alpha} F_{\beta}^{\prime}=F_{\delta}^{\prime} F_{\gamma}\left(\tau_{\gamma \alpha} \otimes \tau_{\delta \beta}^{\prime} \otimes e\right)\left(\varphi_{213} R_{12} \varphi^{-1}\right)
$$

Then the corresponding left covariant multiplets obey braid relations of the form

$$
{ }_{\alpha} F_{\beta} F^{\prime}=\left(\tilde{\tau}_{\alpha \gamma} \otimes \tilde{\tau}_{\beta \delta}^{\prime} \otimes e\right)\left(\varphi_{213} R_{12} \varphi^{-1}\right){ }_{\delta} F^{\prime}{ }_{\gamma} F
$$


4. On covariant products, the transformation (7.20) acts according to

$$
\left.F_{\alpha} \times F_{\beta}^{\prime}\left(\left(\tau_{\alpha \gamma} \otimes \tau_{\beta \delta}^{\prime}\right) \otimes e\right)(w)\right)=\left(\tilde{\tau}_{\delta \beta}^{\prime} \otimes \tilde{\tau}_{\gamma \alpha} \otimes e\right)\left(f_{12} \varphi^{-1}\right)_{\alpha} F{ }_{\beta} F^{\prime},
$$

where $f$ is the element (7.19). Note that the expression on the right hand side of the equation can be regarded as a linear combination of "left covariant products".

We do not want to prove this proposition here. Details can be found in [8]. Especially the last two items are cumbersome. Note that the theorem establishes a complete symmetry between left and right. It implies that it was just a matter of convenience to write all the relations defining $\mathcal{B}$ in terms of the right covariant product. They can all be rewritten in terms of a "left covariant product" and even in a mixed form, where $\varphi_{x}$ appears on the right and $\varphi_{y}$ to the left of the product $U^{I}(i) U^{J}(j)$. In spite of this left-right symmetry, there is now an algebraic difference between components $U_{\alpha}^{I}(i)$ and the "matrix-elements" of a quasi-quantum group valued gauge field. Matrix elements should be identified with ${ }_{a} U_{b}^{I}(i)$ and the latter differ from $U_{a b}^{I}(i)$.

Now we can proceed exactly as in the Hopf-algebra case. We define an anti-homomorphism $\theta$ by $\theta(\xi)=\xi^{*}$ and its action on left covariant elements,

$$
\theta\left({ }_{\alpha} U^{I}(i)\right)=U_{\gamma}^{I}(-i)\left(\tau_{\gamma \beta}^{I,-i} \otimes i d\right)\left(R^{-1}\right) \eta_{\beta \alpha}^{I, i} .
$$

On right covariant elements, $\theta$ acts according to

$$
\theta\left(U_{\alpha}^{I}(i)\right)=v_{I}^{-2} \eta_{\alpha \beta}^{I,-i}\left(\tilde{\tau}_{\gamma \beta}^{I,-i} \otimes i d\right)(R)_{\gamma} U^{I}(-i) .
$$

Although this second formula looks very familiar, it is hard work to derive it from the definition of $\theta$. Consistency with the transformation law is a word by word repetition of the above arguments. To check the compatibility with the braid relations, we start from the equation $(\sqrt{7.29})$ and apply $\theta$. The rest is straightforward. Conjugation with $\kappa \in \mathcal{G}$ improves this anti-automorphism $\theta$ so that it furnishes a $*$-operation $\sigma_{\kappa} \circ \theta$ on $\mathcal{B}$.

The observable algebra $\mathcal{A}$ is obtained as an algebra of invariants again. Let $<U_{\alpha}^{I}(i), \times>$ denote the linear space generated the (right-) covariant product $\times$. Observe that this space is not closed under ordinary associative products in $\mathcal{B}$. Within $\left\langle U_{\alpha}^{I}(i), \times>\right.$ only the quasi-associative multiplication with the covariant product $\times$ is possible. We define $\mathcal{A}$ to be the subspace of invariants, i.e.

$$
\mathcal{A} \equiv\left\{A \in<U_{\alpha}^{I}(i), \times>\mid \xi A=A \xi \text { for all } \xi \in \mathcal{G}\right\} .
$$

Obviously $\mathcal{A}$ is closed under covariant multiplication and since (by proposition 16) the covariant product and the associative product coincide for invariants, $\mathcal{A}$ comes equipped with an associative product. 
Theorem 18 (algebra $\mathcal{A}$ of observables) Let $\mathcal{G} \equiv \otimes \mathcal{G}_{x}$ be a ribbon quasiHopf algebra. Then the the associative algebra $\mathcal{A}$ of invariants in the vector space $\left\langle U_{\alpha}^{I}(i), \times>\right.$ is a ${ }^{*}$-algebra with $*$-operation $A^{*}=\left(\sigma_{k} \circ \theta\right)(A)$. If the quantum dimensions $d_{I}$ are positive and assumption (3.15) is satisfied, the linear functional $\omega: \mathcal{B} \mapsto \mathbf{C}$ defined by (6.6) restricts to a positive linear functional $\omega$ on $\mathcal{A}$, i.e. $\omega\left(A^{*} A\right) \geq 0$ for all $A \in \mathcal{A}$. The algebra $\mathcal{A}$ is independent of the position of eyelashes.

To construct the regular representation one can follow a recipe given in [1]. We do not want to repeat the individual steps here. Instead let us sketch the main results. The analogue of $\mathcal{F}$ is non-associative unital algebra with unit $\Omega$. The generators $u_{\alpha}^{I}(i)$ of $\mathcal{F}$ can be braid commuted with (6.1) (i.e. the braid relations of elements $u_{\alpha}^{I}(i)$ contain no $\varphi$ ). One can prove that eq. (6.2, 6.3) continue to hold. To define a representation $\pi$ of $\mathcal{B}$ on $\mathcal{F}$ one uses rel. (6.4.6.5). This provides us with a notion of covariance and invariance in $\mathcal{F}$ in the same way as before. Invariant elements in $\mathcal{F}$ form an associative subalgebra $\mathcal{F}^{\text {inv }}$. Finally, the functional $\omega$ can be used to define a scalar product on $\mathcal{F}$ as this was done before.

The non-associativity of $\mathcal{F}$ is relatively harmless. As one may guess by now, $\mathcal{F}$ turns out to be quasi-associative in the sense that products with different positions of brackets are linear combinations of each other. The "re-association" can be performed with the help of

$$
\left(\psi_{\alpha} \cdot \psi_{\beta}^{\prime}\right) \cdot \psi_{\gamma}^{\prime \prime}=\psi_{\delta} \cdot\left(\psi_{\rho}^{\prime} \cdot \psi_{\sigma}^{\prime \prime}\right)\left(\tau_{\alpha \delta} \otimes \tau_{\beta \rho}^{\prime} \otimes \tau_{\gamma \sigma}^{\prime \prime}\right)(\varphi),
$$

which hold whenever $\psi_{\alpha}, \psi_{\beta}^{\prime}, \psi_{c}^{\prime \prime}$ transform covariantly according to the representation $\tau, \tau^{\prime}, \tau^{\prime \prime}$ of $\mathcal{G}$. In the title of this subsection we suggest the name "quasi-quantum group" for the algebra generated by the $u_{\alpha}^{I}(i)$ on a given link $i$. Because of the quasi-associativity of the multiplication in $\mathcal{F}$, there is now a dramatic difference between "quasi-quantum groups" and quasi-Hopf algebras. At least for a special choice of the twists $F_{x}$ in the endpoints, the algebra generated by the $u_{\alpha}^{I}(i)$ is dual to the quasi-Hopf algebra $\mathcal{G}_{*}$. Since co-associativity generically fails to hold in $\mathcal{G}_{*}$, this duality gives another "explanation" why the algebra of "functions" on the space of connections becomes quasi-associative.

As a corollary of the above theorem and the remarks we made before, it is finally obtained that

Corollary 19 Hamiltonian Chern Simons theory can be quantized for all integer values of the level $k$ and for every simple Lie algebra $\mathcal{G}$.

This corollary needs some explanation because there are many senses of the word "quantization". Here to quantize is treated in the sense of deformation quantization. Namely, one should construct an algebra of observables supplied with a $*$-operation and a positively defined trace functional. Consistence with the Poisson brackets is required in the classical limit. In this sense we obtained 
a quantization of the algebra of lattice gauge observables. In order to make the same statement about the whole Hamiltonian Chern Simons theory, we should impose the quantum flatness conditions in a way consistent with the $*$-operation. We postpone this question to the next paper.

\subsection{Twist equivalence}

We are now prepared to show that the algebra of observables actually does only depend on the "twist class" of $\mathcal{G}_{x}$. Let us make this statement precise. Suppose that $\hat{\mathcal{G}}_{x}$ is a second set of quasitriangular quasi Hopf-*-algebras which are again twist equivalent to the $\mathcal{G}_{*}$ but with possibly different twist elements $\hat{F}_{x} \in \mathcal{G}_{*}$. Then one can follow all the steps described above to build a lattice algebra $\hat{\mathcal{B}}$ with *-operation $*$, an algebra of invariants $\hat{\mathcal{A}}$ etc.. In general these structures will depend on the choice of $\mathcal{G}_{x}$. The observable part of the theory, however, does not change.

Theorem 20 (twist independence of $\mathcal{A})$ Suppose that $\left(\mathcal{G}_{x}\right)_{x \in S}$ and $\left(\hat{\mathcal{G}}_{x}\right)_{x \in S}$ are two families of gauge symmetries and that $\mathcal{G}_{x}$ as well as $\hat{\mathcal{G}}_{x}$ are twist equivalent to the quasitriangular quasi Hopf-*-algebra $\mathcal{G}_{*}$ for all $x \in S$. Then there is a *-isomorphism $i: \hat{\mathcal{A}} \mapsto \mathcal{A}$.

Proof: We denote the ratio of the twist elements by $h_{x}=F_{x}^{-1} \hat{F}_{x}$. With the help of the isomorphism $\iota_{x}$ this family is lifted to an element $h \in \mathcal{G} \otimes \mathcal{G}$. There is an isomorphism $i: \hat{\mathcal{B}} \mapsto \mathcal{B}$ defined by $i(\xi)=\iota_{x}^{-1} \circ \hat{\iota}_{x}(\xi)$ for all $\xi \in \hat{\mathcal{G}}_{x}$ and

$$
i\left(\hat{U}_{\alpha}^{I}(i)\right)=U_{\beta}^{I}(i)\left(\tau_{\beta \alpha}^{I, i} \otimes i d\right)(h)
$$

With all the experience we gained in prior calculations, the homomorphism property is now almost obvious. So let us directly proceed to the consistency with $*$. To prove that $i$ is a ${ }^{*}$-homomorphism, one has to know, how the element $w$ behaves under twists. The answer is

$$
w=\Delta\left(h_{\sigma}^{2}\right) h(i \otimes i)(\hat{w})\left(\mathcal{S}^{-1}\left(h_{\sigma}^{1}\right) \otimes e\right), \quad h=\sum h_{\sigma}^{1} \otimes h_{\sigma}^{2} .
$$

Let us agree from now on to identify elements in $\mathcal{G}$ and $\hat{\mathcal{G}}$ with the help of the isomorphism $i$, so that, for example, $\hat{w}$ can be regarded as an element in $\hat{\mathcal{G}} \otimes \hat{\mathcal{G}}$ without the extra action of $(i \otimes i)$. The twist dependence of $w$ yields the action of $i$ on left-covariant elements.

$$
i\left({ }_{\alpha} \hat{U}^{I}(i)\right)=\left(\tilde{\tau}_{\alpha \beta}^{i} \otimes i d\right)\left(h^{-1}\right){ }_{\beta} U^{I}(i)
$$

The element $\eta$ is independent of the twist, and consequently $\hat{\eta}^{I, i}$ and $\eta^{I, i}$ can be identified in the following calculation

$$
i\left(\hat{\theta}\left({ }_{\alpha} \hat{U}^{I}(i)\right)\right)=U_{\gamma}^{I}(-i)\left(\tau_{\gamma \beta}^{I,-i} \otimes i d\right)\left(h \hat{R}^{-1}\right) \eta_{\beta \alpha}^{I, i}
$$




$$
\begin{aligned}
& =U_{\gamma}^{I}(-i)\left(\tau_{\gamma \beta}^{I,-i} \otimes i d\right)\left(R^{-1} h^{\prime}\right) \eta_{\beta \alpha}^{I, i} \\
& =\theta\left({ }_{\delta} U^{I}(i)\right)\left(\tilde{\tau}_{\delta \alpha}^{I, i} \otimes i d\right)\left(h^{\prime}\right) \\
& =\theta\left(\left(\tilde{\tau}_{\alpha \delta}^{I, i} \otimes i d\right)\left(h^{-1}\right){ }_{\delta} U^{I}(i)\right)=\theta\left(i\left({ }_{\alpha} U^{I}(i)\right) .\right.
\end{aligned}
$$

So $i$ is compatible with the anti-automorphisms $\theta, \hat{\theta}$ and hence with the *operations on $\mathcal{B}, \hat{\mathcal{B}}$. The proof of twist independence is complete if we can show that $i$ restricts to a map between the observable algebras $\hat{\mathcal{A}}$ and $\mathcal{A}$. To see this we recall that observables are linear combinations of elements

$$
\hat{U}_{\alpha_{n}}^{I_{n}}\left(i_{n}\right) \hat{\times}\left(\ldots \hat{\times}\left(\hat{U}_{\alpha_{2}}^{I_{2}}\left(i_{2}\right) \hat{\times} \hat{U}_{\alpha_{1}}^{I_{1}}\left(i_{1}\right)\right) \ldots\right) \hat{C}_{\alpha_{n}, \ldots \alpha_{2}, \alpha_{1}},
$$

where $\hat{C}$ is supposed to possess the usual intertwining properties,

$$
\left(\tau^{I_{n}, i_{n}} \hat{\otimes}\left(\ldots \hat{\otimes}\left(\tau^{I_{2}, i_{2}} \hat{\otimes} \tau^{I_{1}, i_{1}}\right) \ldots\right)\right)(\xi) \hat{C}=\hat{C} \epsilon(\xi)
$$

for all $\xi \in \hat{G}$. To evaluate $i$ on such elements one uses the following compatibility of $i$ with covariant multiplication

$$
\begin{aligned}
i\left(\hat{U}_{\alpha}^{I}(i) \hat{\times} \hat{U}_{\beta}^{J}(j)\right) & =U_{\gamma}^{I}(i) U_{\sigma}^{J}(j)\left(\tau_{\gamma \alpha}^{I, i} \otimes \tau_{\sigma \beta}^{J, j} \otimes e\right)\left((i d \otimes \Delta)(h) h_{23} \hat{\varphi}\right) \\
& =U_{\gamma}^{I}(i) U_{\sigma}^{J}(j)\left(\tau_{\gamma \alpha}^{I, i} \otimes \tau_{\sigma \beta}^{J, j} \otimes e\right)\left(\varphi h_{12}(\hat{\Delta} \otimes i d)(h)\right) \\
& =U_{\gamma}^{I}(i) \times U_{\sigma}^{J}(j)\left(\tau_{\gamma \alpha}^{I, i} \otimes \tau_{\sigma \beta}^{J, j} \otimes i d\right)\left(h_{12}(\hat{\Delta} \otimes i d)(h)\right) .
\end{aligned}
$$

When we act on multiple $\times$-products we obtain a similar expression in which the argument of $\left(\tau^{I_{n}, i_{n}} \otimes \ldots \otimes \tau^{I_{1}, i_{1}} \otimes i d\right)$ splits into two factors. The right factor essentially annihilates by the multiplication with $\hat{C}$ while the left factor is trivial in the last component and consequently gives rise to a complex linear combination of

$$
U_{\alpha_{n}}^{I_{n}}\left(i_{n}\right) \times\left(\ldots \times\left(U_{\alpha_{2}}^{I_{2}}\left(i_{2}\right) \times U_{\alpha_{1}}^{I_{1}}\left(i_{1}\right)\right) \ldots\right) \hat{C}_{\alpha_{n}, \ldots \alpha_{2}, \alpha_{1}} .
$$

Since the image $i(G)$ of an invariant element $G \in \hat{\mathcal{B}}$ is automatically invariant, this proves the proposition.

\section{Outlook}

In the first part of our work we discussed a new notion of quantum group lattice gauge models. The algebra of observables in such a model has been constructed. Our definitions were motivated by the Fock-Rosly discussion of classical Chern Simons theories. From our initial remarks on this topic it is almost clear that there is a deep connection between quantized Chern Simons theories and our lattice gauge models. Let us sketch here the main points that we are going to discuss in details in the second paper devoted to the same subject. 
The first important step towards the quantum Chern-Simons theory is to implement the flatness condition in the lattice gauge theory. The idea is the following. Let us introduce a monodromy matrix for each plaquette so that

$$
M=U\left(i_{1}\right) \ldots U\left(i_{s}\right),
$$

where the links $i_{1} \ldots i_{s}$ surround the chosen plaquette. Our purpose is to fix somehow the eigenvalues of the matrix $M$ as we did in Section 2 (see formulae $(2.23,2.24))$. From the very beginning it is not clear that there exists a quantum analogue of this procedure. It might happen that operators corresponding to eigenvalues of $M$ do not commute and it would cause serious problems. Fortunately, these operators commute with each other and moreover they belong to the center of the algebra of observables. So one can find a set of central projectors which efficiently fix eigenvalues of monodromy matrices for all elementary plaquettes. One of such projectors has been introduced in [5] and called quantum $\delta$-function. Having imposed the condition of flatness we obtain the quantum algebra of functions on the moduli space of flat connections (moduli algebra) or, in other words, the algebra of observables of the Hamiltonian Chern-Simons theory. The moduli algebra enjoys an important property. It does not depend on the graph on the Riemann surface which we have started with [6]! It is possible to prove that the moduli algebras constructed starting from different graphs are canonically isomorphic to each other. Thus, the algebra of observables is defined by the symmetry algebra, Riemann surface with marked points and by the set of representations of the symmetry algebra assigned to the marked points. After the graph independence of the construction is established we have a good chance to prove that the lattice gauge model defines the same theory as the continuous Chern Simons model.

The important question which we face at this stage is how to construct the representation theory of the moduli algebra. There are two different approaches to this problem. The first of them (let us call it algebraic) is based on the graph independence of the moduli algebra. One can choose the simplest possible graph and consider the moduli algebra in some explicit coordinates given by the algebra of graph connections on this chosen graph. For generic values of the deformation parameter $q$ this method has been applied in [21]. There the graph is chosen to be a bunch of circles on a Riemann surface of genus $g$ with $n$ marked points. The first $g$ circles represent $a-$ and $b$-cycles winding around the handles of the surface and $n$ cycles which surround the marked points. Using this particular graph one can describe the representation theory of the moduli algebra quite efficiently. We shall fulfill this program for $q$ being a root of unity.

The second approach to the representation theory (let us call it geometric) is based on two simple observations. First of all we already know the desirable answer. The algebra of observables of the Hamiltonian Chern-Simons theory must act in the Hilbert space of this theory. The latter is isomorphic to the space of conformal blocks in the corresponding WZNW model or, more technically, to 
the space of solutions of the Knizhnik-Zamolodchikov equation satisfying certain conditions [13, 29]. The second observation concerns the nature of structure constants which we use to define the algebra of graph connections. Basically we use two objects, $R$-matrix and the associator $\varphi$. Both of them may be regarded as certain monodromy matrices for solutions of the Knizhnik-Zamolodchikov equation [11. These two facts enable us to represent the observable algebra directly in the space of conformal blocks. Particular operators act as combinations of monodromies of the Knizhnik-Zamolodchikov equation. Let us mention that the idea of the geometric approach is essentially borrowed from the combinatorial description of Vassiliev-Kontsevich knot invariants [18], [23].

The geometric construction of the representation theory of the moduli algebra will provide the representations which are realized directly in the Hilbert space of the continuous Chern Simons theory. This will be a final check of the conjecture that the lattice gauge model presented in this paper indeed coincides with the Hamiltonian Chern Simons theory.

The ideas which we have shortly described in this Section will be considered in details in the forthcoming paper.

\section{Acknowledgments}

This work was initiated while two of us (A.A.,V.S.) stayed at the Erwin Schrödinger International Institute in Vienna. We would like to thank the local organizers for providing ideal working conditions and a pleasant atmosphere. In the initial phase, V. V. Fock contributed through intense discussions with one of the authors (A.A.). Conversations with A. Beilinson, V.G. Drinfeld, A. Jaffe, T. Kerler, A. Lesniewski, G. Mack, F. Nill and K. Szlachanyi are gratefully acknowledged.

The work of V.S. was partly supported by the Department of Energy under DOE Grant No. DE-FG02-88ER25065. A.A. was supported by the Swedish Natural Science Research Council (NFR) under the contract F-FU 06821-304. The work of H.G. was done in the framework of the project P8916-PHY of the 'Fonds zur Förderung der wissenschaftlichen Forschung in Österreich'.

\section{References}

[1] G. Mack, V. Schomerus, Action of truncated quantum groups on quasi quantum planes and a quasi-associative differential geometry and calculus, Commun. Math. Phys. 149, (1992), 513

[2] G. Mack, V. Schomerus, Quasi Hopf quantum symmetry in quantum theory, Nucl. Phys. B370(1992),185 
[3] A. Sudbury, Non-commuting coordinates and differential operators in: Quantum groups, T. Curtright et al. (eds), World Scientific, Singapore 1991

[4] A.N.Kirillov,N. Reshetikhin, Representations of the algebra $U_{q}(s l(2))$, qorthogonal polynomials and invariants of links, preprint LOMI E-988,Leningrad 1988

[5] D.V. Boulatov, q-deformed lattice gauge theory and three manifold invariants, Int. J. Mod. Phys. A8,(1993), 3139

[6] V.V. Fock, A.A. Rosly, Poisson structures on moduli of flat connections on Riemann surfaces and r-matrices, preprint ITEP 72-92, June 1992, Moscow

[7] V. Schomerus, Quantum symmetry in quantum theory, PhD thesis, DESY $-93-18$

[8] V. Schomerus, Construction of field algebras with quantum symmetry from local observables, Commun. Math. Phys., to appear

[9] V. Schomerus, Quasi quantum group covariant q-oscillators, Nucl. Phys. B401, (1993), 455

[10] V.G. Drinfel'd, Quantum groups, Proc. ICM (1987) 798

[11] V.G. Drinfel'd, Quasi Hopf algebras and Knizhnik Zamolodchikov equations, in: Problems of modern quantum field theory, Proceedings Alushta 1989, Research reports in physics, Springer Verlag Heidelberg 1989

V.G. Drinfel'd, Quasi-Hopf algebras, Leningrad. Math. J. Vol. 1 (1990), No. 6

[12] M. Scheunert, The antipode of and the star operation in a Hopf algebra, J. Math. Phys. 34, (1993), 320

[13] E.Witten, Quantum field theory and the Jones polynomial, Commun. Math. Phys. 121, (1989),351

[14] S. Elitzur,G. Moore, A. Schwimmer, N. Seiberg, Remarks on the canonical quantization of the Chern-Simons-Witten theory, Nucl. Phys. 326, (1989), 108

[15] A. Alekseev, L. Faddeev, S. Shatashvili Quantization of symplectic orbits of compact Lie groups by means of the functional integral Geom. and Phys., vol.5, no.3, 1989, pp.391-406.

[16] S.Axelrod, I.M.Singer, Chern-Simons Perturbation Theory II, preprint hepth/9304087. 
[17] A.Gerasimov, Localization in $G W Z W$ and Verlinde formula, Uppsala preprint hep-th/9305090.

[18] S.Piunikhin, Combinatorial expression for universal Vassiliev link invariant, Harvard preprint hep-th/9302084.

[19] A.Yu.Alekseev, L.D.Faddeev, M.A.Semenov-Tian-Shansky Hidden Quantum groups inside Kac-Moody algebras, Commun. Math. Phys. 149, no.2 (1992) p.335.

[20] D.Bar-Natan, E.Witten Perturbative Expansion of Chern-Simons Theory with Non-Compact Gauge Group, Commun. Math. Phys. 141, (1991) p.423.

[21] A.Yu.Alekseev, Integrability in the Hamiltonian Chern-Simons theory, Uppsala preprint hep-th/9311074, to appear in St.-Petersburg. Math.J.

[22] D. Altschuler, A. Coste Quasi-quantum groups, knots, three manifolds and topological field theory, Commun. Math. Phys. 150 (1992) 83

[23] P. Cartier, Construction Combinatoire des Invariants de Vassiliev-Kontsevich des Nœeds, preprint of the Erwin Schrödinger Institute ESI 15 (1993), Vienna, Austria.

[24] V.G. Turaev, O.Y. Viro, State sum of 3-manifolds and quantum 6j-symbols, LOMI preprint (1990)

[25] B. Durhuus, H.P. Jakobsen, R. Nest, A construction of topological quantum field theories from 6j-symbols, Nucl. Phys. B (Proc. Suppl.) (1991), 666

[26] M. Karowski, R. Schrader, A combinatorial approach to topological quantum field theories and invariants of graphs, Commun. Math. Phys. 151, (1993), 355

M. Karowski, R. Schrader, A quantum group version of quantum gauge theories in two dimensions, J. Phys. A 25, (1992), L1151

[27] E. Witten, On quantum gauge theories in two dimensions, Commun. Math. Phys. 141, (1991), 153

[28] N. Reshetikhin, V. G. Turaev, Ribbon graphs and their invariants derived from quantum groups, Commun. Math. Phys. 127, (1990),1

[29] J. Fröhlich, C. King, The Chern Simons theory and knot polynomials, Commun. Math. Phys. 126 (1989) 167 